\title{
DCore: Integrated DMFT software for correlated electrons
}

\author{
Hiroshi Shinaoka $^{1 *}$, Junya Otsuki ${ }^{2}$, Mitsuaki Kawamura ${ }^{3}$, Nayuta Takemori ${ }^{2}$, \\ Kazuyoshi Yoshimi ${ }^{3}$ \\ 1 Department of Physics, Saitama University, Saitama 338-8570, Japan \\ 2 Research Institute for Interdisciplinary Science, Okayama University, Okayama 700-8530, \\ Japan \\ 3 Institute for Solid State Physics, University of Tokyo, Chiba 277-8581, Japan \\ * shinaoka@mail.saitama-u.ac.jp
}

July 25, 2021

\begin{abstract}
We present a new open-source program, DCore, that implements dynamical mean-field theory (DMFT). DCore features a user-friendly interface based on text and HDF5 files. It allows DMFT calculations of tight-binding models to be performed on predefined lattices as well as ab initio models constructed by external density functional theory codes through the Wannier90 package. Furthermore, DCore provides interfaces to many advanced quantum impurity solvers such as quantum Monte Carlo and exact diagonalization solvers. This paper details the structure and usage of DCore and shows some applications.
\end{abstract}

\section{Contents}

$\begin{array}{lll}1 & \text { Introduction } & 2\end{array}$

2 Methodology 3

2.1 Model 3

2.2 DMFT formalism 4

2.3 Block structure of Green's functions

2.4 Supported functionality 4

3 Structure of DCore and flowchart of simulation 5

4 Format of input and output files 6

4.1 Input file format 6

4.2 Output-file format 9

5 Installation $\quad 10$

6 Examples $\quad$ 10

6.1 First example: Square-lattice Hubbard model 11

6.1.1 Model construction 11

6.1.2 Self-consistent calculations 11 
6.1.3 Dynamical quantities 14

6.1.4 CT-QMC solver 15]

6.1.5 Wannier90 interface 17

6.1.6 Antiferromagnetic state 18

6.2 Solving multi-orbital model using QMC solver: $t_{2 \mathrm{~g}}$ model on a Bethe lattice 19

6.2.1 Model definition 20

6.2.2 DMFT calculation 21

6.3 DFT+DMFT exmaple: $\mathrm{SrVO}_{3} \quad 22$

6.3.1 Construction of Wannier functions 23

6.3.2 Model definition 23

6.3.3 Parameters for self-consistent calculations and post processing 24

6.3.4 Results 24]

7 Conclusion

\section{Introduction}

Dynamical mean-field theory (DMFT) has become a standard theoretical tool for studying strongly correlated electronic systems [1]. In a DMFT calculation, an original lattice model is mapped to an effective Anderson impurity problem whose bath degrees of freedom are self-consistently determined. Although the DMFT formalism was originally proposed for tight-binding models such as Hubbard models, it can be combined with density functional theory (DFT) based on ab initio calculations to describe the electronic properties of strongly correlated materials [2]. This composite framework, called DFT+DMFT, has been applied to various types of materials, such as cuprates, Fe-based superconductors, and $f$-electron materials.

To make DFT+DMFT available to more users and to promote the development of a community of users and developers, there is a strong need for an open-source program package with a user-friendly interface. Some open-source software packages for performing DFT+DMFT calculations have been developed. The TRIQS project aims to provide the necessary components to implement DFT+DMFT codes, including a quantum Monte Carlo (QMC) impurity solver [3] and an interface to DFT codes [4]. The user can implement their own DFT code using the building blocks in Python. The ALPS project provides a DMFT code for simple Hubbard models [5] and implementations of several continuous-time QMC (CT-QMC) impurity solvers 6 6]. w2dynamics 9 provides a state-of-the-art implementation of a QMC algorithm and includes a Python program for performing simple DFT+DMFT calculations. DMFTwDFT features a user-friendly interface and has interfaces to various DFT codes and the CT-QMC impurity solver 10. DFT+embedded DMFT Functional (eDMFT) 11 features a full set of DFT+DMFT functionalities but it is not open-source software.

DCore is an open-source program package that implements (DFT+)DMFT calculations for multi-orbital systems. This package is built on top of existing TRIQS Python libraries and provides interfaces to external impurity solvers from the ALPS [6,7] and TRIQS projects [3.12] as well as exact-diagonalization solvers based on pomerol [13] and $H \Phi[14]$. DCore provides a flexible interface based on text and HDF5 files and does not require extensive expert knowl- 
edge. It also provides a set of command line tools to analyze a self-consistent solution and evaluate physical quantities such as momentum-resolved spectral functions.

The remainder of this paper is organized as follows. In Sec. 2, the methods used for implementing DCore are described. In Sec. 3, the structure of DCore and a flowchart of simulations are shown. In Sec. 4, the formats of input and output files are explained. In Sec. 5 , brief instructions on the installation of DCore are provided. In Sec. 6, several run examples of DCore from simple Hubbard models to DFT+DMFT calculations for a correlated material are demonstrated. In Sec. 7, a summary and the conclusions of this paper are given.

\section{Methodology}

In this section, a brief explanation of the methodology used for implementing DCore is provided. For more technical details of the DFT+DMFT method, we refer the reader to a review article 2]. The current version of DCore implements only one-shot DFT+DMFT calculations based on Wannier9 $\left[{ }^{1}\right.$ and does not support charge self-consistent calculations. In other words, it specializes in the analysis of multi-orbital Hubbard models, making it different from eDMFT and DMFTwDFT.

\section{$2.1 \quad$ Model}

In DCore, the Hamiltonian on the basis of a tight-binding model is given as follows:

$$
\begin{aligned}
\mathcal{H} & =\sum_{\boldsymbol{k}=1}^{N} \sum_{i, j \in \mathrm{crsh}} H_{i j}^{\alpha \beta}(\boldsymbol{k}) c_{\boldsymbol{k} i \alpha}^{\dagger} c_{\boldsymbol{k} j \beta}+\sum_{\boldsymbol{R}, i \in \mathrm{crsh}} \mathcal{H}_{\mathrm{int}}(\boldsymbol{R}, i), \\
& =\sum_{\boldsymbol{R}, \boldsymbol{R}^{\prime}}^{N} \sum_{i, j \in \mathrm{crsh}} H_{i j}^{\alpha \beta}\left(\boldsymbol{R}-\boldsymbol{R}^{\prime}\right) c_{\boldsymbol{R} i \alpha}^{\dagger} c_{\boldsymbol{R}^{\prime} j \beta}+\sum_{\boldsymbol{R}, i \in \mathrm{crsh}} \mathcal{H}_{\mathrm{int}}(\boldsymbol{R}, i),
\end{aligned}
$$

where the first term in Eq. (1) denotes a non-interacting Hamiltonian. Here, $i$ and $j$ run over both correlated (crsh) and uncorrelated "shells" in a unit cell. $\alpha$ and $\beta$ denote the spin-orbital

index in each shell. $c_{\boldsymbol{k} i \alpha} / c_{\boldsymbol{k} i \alpha}^{\dagger}$ indicates an annihilation/creation fermion operator at the $i$-th shell with momentum $\boldsymbol{k}$. $N$ denotes the number of $\boldsymbol{k}$ points. The second term $\mathcal{H}_{\text {int }}(\boldsymbol{R}, i)$ denotes an interaction at the $i$-th correlated shell. Here, $\boldsymbol{R}$ denotes the coordinates of a unit cell. This Hamiltonian is given as

$$
\mathcal{H}_{\text {int }}(\boldsymbol{R}, i)=\frac{1}{2} \sum_{\alpha \beta \gamma \delta} U_{\alpha \beta \gamma \delta}(i) c_{\boldsymbol{R} i \alpha}^{\dagger} c_{\boldsymbol{R} i \beta}^{\dagger} c_{\boldsymbol{R} i \delta} c_{\boldsymbol{R} i \gamma},
$$

where $U_{\alpha \beta \gamma \delta}(i)$ denotes a spin-full four-rank Coulomb tensor. Note that $\mathcal{H}_{\text {int }}$ is limited to intra-shell interactions.

\footnotetext{
${ }^{1}$ DCore uses a Wannier90 converter in TRIQS/DFTTools.
} 


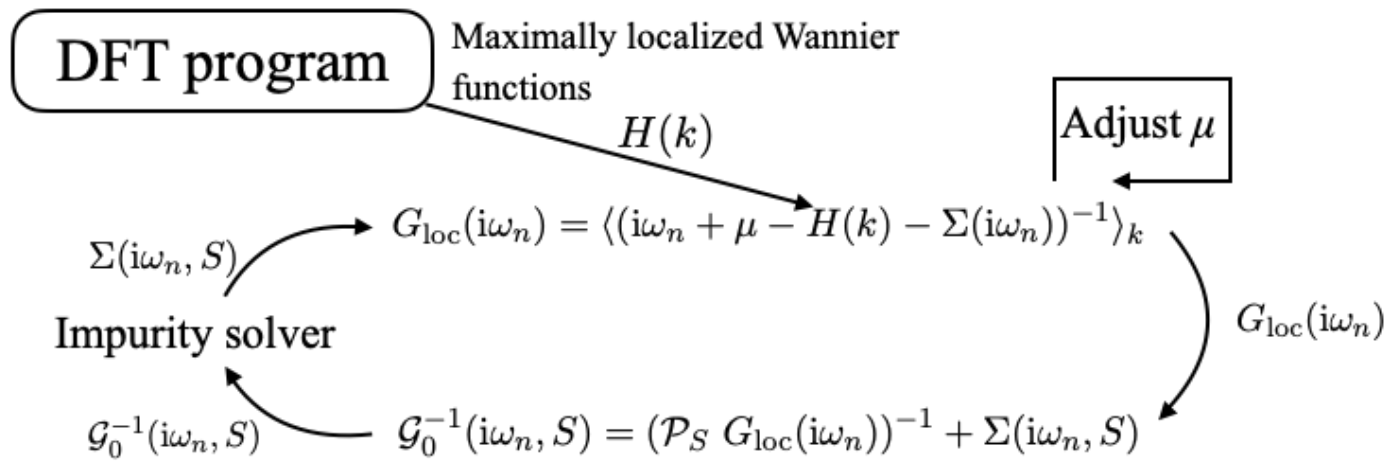

Figure 1: Illustration of the DMFT self-consistency cycle defined in Eqs. (4)-(6). The most numerically expensive step is the solution of the impurity problem, i.e., the calculation of the self-energy for a given $\mathcal{G}_{0}^{-1} .\langle\ldots\rangle$ denotes an average over the momentum space and $\mu$ is the chemical potential.

\subsection{DMFT formalism}

DCore solves the following DMFT self-consistent equations:

$$
\begin{aligned}
G_{\text {loc }}\left(\mathrm{i} \omega_{n}\right) & =\left\langle\left[\mathrm{i} \omega_{n}+\mu-H(k)-\Sigma\left(\mathrm{i} \omega_{n}\right)\right]^{-1}\right\rangle_{k}, \\
\mathcal{G}_{0}^{-1}\left(\mathrm{i} \omega_{n}, S\right) & =\left[\mathcal{P}_{S} G_{\text {loc }}\left(\mathrm{i} \omega_{n}\right)\right]^{-1}+\Sigma\left(\mathrm{i} \omega_{n}, S\right), \\
\Sigma\left(\mathrm{i} \omega_{n}, S\right) & =\text { New self-energy from an external impurity solver. }
\end{aligned}
$$

Here, $\omega_{n} \equiv(2 n+1) \pi T$ is a fermionic Matsubara frequency at temperature $T,\langle\cdots\rangle$ denotes an average over the momentum space, $S$ indexes correlated shells, and $\mathcal{P}_{S}$ is the projector to the $S$-th correlated shell. Green's functions $G_{\mathrm{loc}}\left(\mathrm{i} \omega_{n}\right)$ and $\mathcal{G}_{0}\left(\mathrm{i} \omega_{n}\right)$ and the hybridization function $\Delta\left(\mathrm{i} \omega_{n}\right)$ are matrices, and their inversions are regarded as inverse matrices. The chemical potential $\mu$ is either adjusted to obtain a desired number of electrons at each iteration or is fixed at a given value. Some QMC impurity solvers accept the hybridization function defined below as an input:

$$
\Delta\left(\mathrm{i} \omega_{n}, S\right)=\left(\mathrm{i} \omega_{n}+\mu\right) I-\mathcal{P}_{S}\langle H(k)\rangle_{k}-\mathcal{G}_{0}^{-1}\left(\mathrm{i} \omega_{n}, S\right),
$$

where $I$ is the identity matrix.

\subsection{Block structure of Green's functions}

The Green's functions are assumed to be either spin-diagonal or spin-off-diagonal. The former is sufficient for computing a paramagnetic state or a collinear magnetic state polarized along the $z$ axis. The latter allows $\Sigma\left(\mathrm{i} \omega_{n}\right), G\left(\mathrm{i} \omega_{n}\right)$, and $\mathcal{H}(k)$ to have spin-off-diagonal elements. This must be used when $\mathcal{H}$ has spin-orbit coupling that mixes the spin-up/down sectors or when one computes non-collinear magnetic states.

\subsection{Supported functionality}

DCore offers a lot of functionality that is useful in practical calculations, including the following. 
- Non-collinear magnetism

- Symmetrization of the self-energy according to rotational groups

- Copying the self-energy between equivalent shells

- Several methods for double-counting corrections

\section{Structure of DCore and flowchart of simulation}

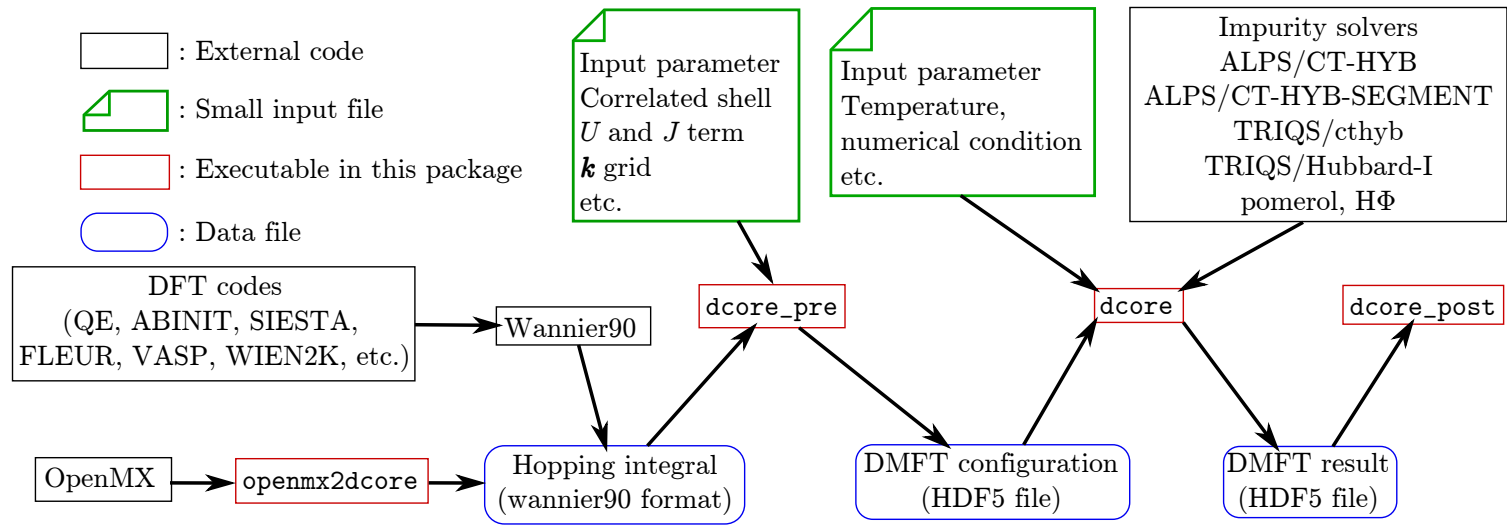

Figure 2: Structure of DCore.

DCore contains a set of programs that perform DMFT calculations for models and materials. The structure of the programs and data flow is summarized in Fig. 2 .

DCore consists of three layers: (i) interface layer, (ii) DMFT self-consistent loop, and (iii) post-processing. These are respectively performed by the executables dcore_pre, dcore, and dcore_post. Input parameters are provided by a single text file, which is read by all three programs. Data generated by dcore_pre and dcore are separately stored in an HDF5 file and passed to the next process.

(i) Interface layer: dcore_pre dcore_pre generates the HDF5 file necessary for the DMFT loop. Users specify parameters that define a model, such as the hopping parameters on a certain lattice, and interactions. The hopping parameters are given either for preset models (e.g., square lattice, Bethe lattice) or using the Wannier90 format.

(ii) DMFT self-consistent loop: dcore

dcore is the main program for the DMFT calculations. The effective impurity problem is solved repeatedly to satisfy the self-consistency condition of the DMFT. For solving the impurity problem, dcore calls an external program. As an external program, we can select ALPS/CT-HYB [6], ALPS/CT-HYB-SEGMENT [7, or TRIQS/cthyb [3] as a CT-QMC solver, TRIQS/Hubbard-I [12] as a Hubbard-I solver, or pomerol [13] or $\mathrm{H} \Phi$ 14 as an exact diagonalization (ED) solver. 
(iii) Post-processing: dcore_post dcore_post computes some physical quantities from the converged solution of the DMFT loop. Currently, the (projected) density of states and correlated band structures (momentumresolved single-particle excitation spectrum) can be calculated.

\section{Format of input and output files}

In this section, the format of input and output files for DCore is briefly explained.

\subsection{Input file format}

The input file of DCore consists of six parameter blocks, respectively named [model], [system], [impurity_solver], [control], [tool], and [mpi]. The format of the DCore input file is the same for all programs but the included blocked depend on the program, as shown in Table 1, Each block is described below.

\begin{tabular}{lcccc}
\hline Block & dcore_pre & dcore & dcore_check & dcore_post \\
\hline model & Yes & Yes & Yes & Yes \\
system & - & Yes & Yes & Yes \\
impurity_solver & - & Yes & - & Yes \\
control & - & Yes & - & - \\
tool & - & - & Yes & Yes \\
mpi & - & Yes & - & Yes \\
\hline
\end{tabular}

Table 1: Blocks to be used for each program in DCore.

1. [model] block

This block includes parameters for defining a model to be solved. The parameter types are divided into four parts: (i) Basic parameters, (ii) Lattice parameters, (iii) Interaction parameters, and (iv) Local potential parameters. In the following, we describe the parameters defined in each part.

(i) Basic parameters

There are four basic parameters, namely seedname, nelec, norb, and spin_orbit. seedname determines the name of the model HDF5 file. nelec and norb specify the number of electrons per unit cell and the number of orbitals, respectively. spin_orbit specifies whether the model has spin-orbit interactions.

(ii) Lattice parameters

In DCore, chain, square, cubic, and bethe lattices are prepared as predefined models, as shown in Fig. 3. We can select a lattice type using the lattice parameter and set values of transfer integrals using parameters $t$ and $t$ '. For treating more realistic cases, such as DFT+DMFT calculations, hopping parameters in the Wannier90 format can be imported by selecting wannier90. In this mode, we can set a number of correlated shells in a unit cell and a mapping from correlated shells to equivalent shells using the ncor and corr_to_inequiv parameters, respectively. Figure 4 shows a schematic diagram of the shell structure in wannier90 mode. For experts, the lattice data can be custom prepared using the external mode. In this mode, all necessary data should be directly 
(a) chain

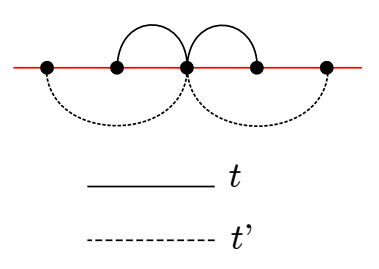

(b) square

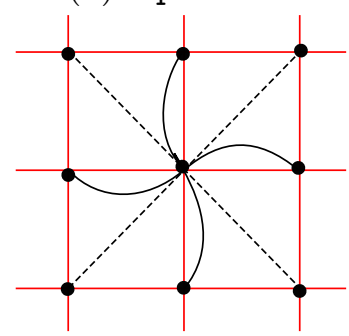

(c) cubic

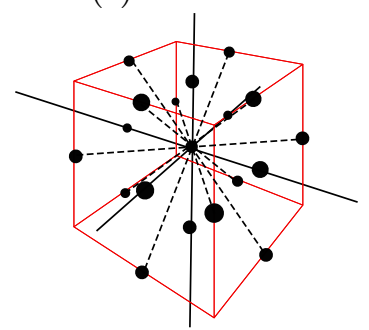

(d) bethe

Density of states

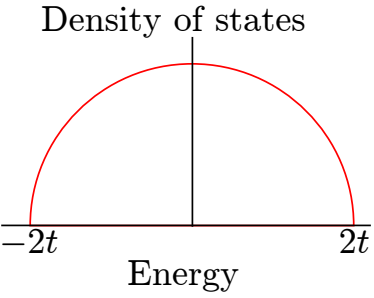

Figure 3:

Predefined lattices in DCore. In the (a) chain, (b) square, and (c) cubic lattices, the solid (dotted) bonds indicate the transfer integrals $t\left(t^{\prime}\right)$. For the $(\mathrm{d})$ bethe lattice, a semicircular density of states with energy ranges $[-2 t: 2 t]$ is defined.

made in the dft_input group of the model HDF5 file. For details, see the reference manual of DFTTools. The information of reciprocal lattice vectors and the number of wave vectors can be also specified.

(iii) Interaction parameters

The interaction part of the Hamiltonian is defined as Eq. (3). The interaction matrix $U_{\alpha \beta \gamma \delta}(i)$ is specified by the parameter interaction. In DCore, three types of interaction, namely (a) kanamori, (b) slater_f, and (c) slater_uj, are defined.

(a) If interaction $=$ kanamori, the Kanamori-type interaction is used; i.e., $U_{\alpha \alpha \alpha \alpha}=$ $U, U_{\alpha \beta \alpha \beta}=U^{\prime}, U_{\alpha \beta \beta \alpha}=J, U_{\alpha \alpha \beta \beta}=J$, where $\alpha \neq \beta$. These parameters at each correlated shell are specified by the parameter kanamori as

interaction $=$ kanamori

kanamori $=\left[\left(U_{-} 1, U^{\prime} \_1, J_{-} 1\right),\left(U_{-} 2, U^{\prime} \_2, J_{-} 2\right), \ldots\right]$.

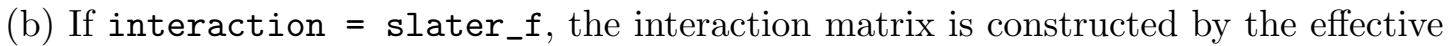
Slater integrals $F_{0}, F_{2}, F_{4}, F_{6}$. These Slater integrals and the angular momentum $l$ at each correlated shell are specified by the parameter slater_f as

interaction $=$ slater_f

slater_f $=\left[\left(\right.\right.$ angular_momentum, F_0, F_2 $\left.\left., F_{-} 4, F_{-} 6\right), \ldots\right]$

It is noted that $F_{0}, F_{2}, F_{4}, F_{6}$ must all be specified.

(c) If interaction $=$ slater_uj, the Slater-type interaction is used. When $U, J$, and the angular momentum $l$ are set, the effective Slater integral is calculated internally in DCore according to the formula defined in Table 2. The $U, J$, and $l$ at each correlated shell are specified by the parameter slater_uj as

interaction $=$ slater_uj

slater_uj $=[($ angular_momentum1, U1, J1), (angular_momentum2, U2, J2), ... ]

(iv) Local potential parameters

An arbitrary local potential can be implemented using parameters local_potential_matrix 
(a) spin_orbit=False (default)

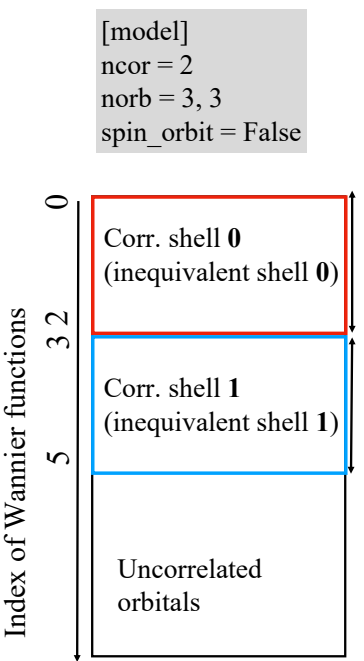

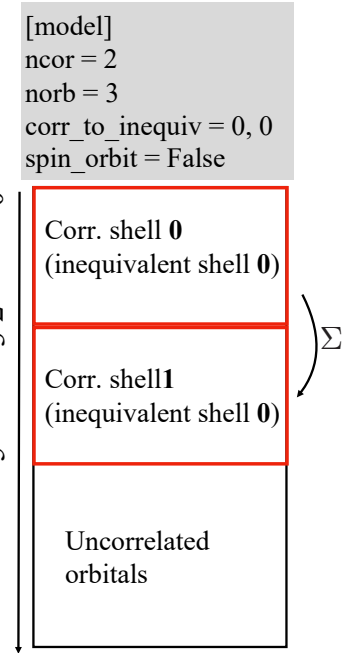

(b) spin orbit $=$ True

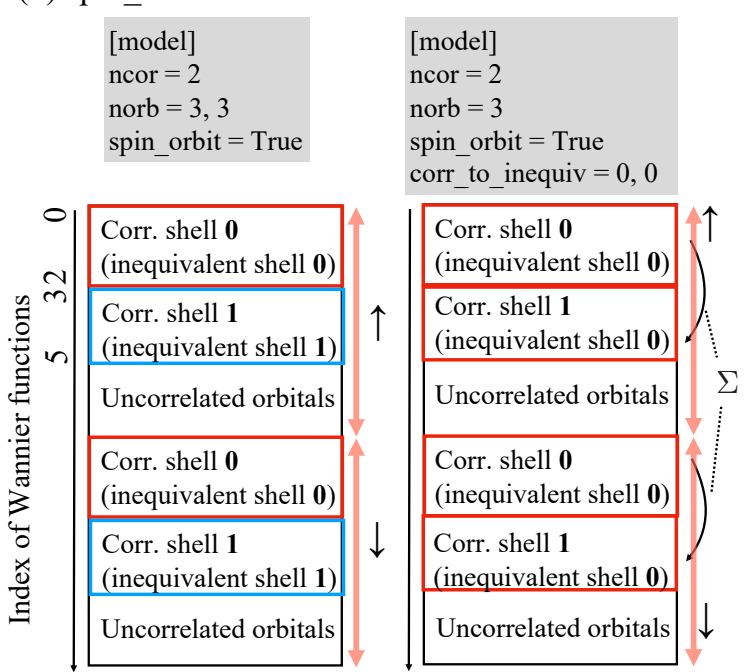

Figure 4: Shell structure of Wannier90 file with (a) spin_orbit=True and (b) spin_orbit=False. As an example, we consider cases with atoms in a unit cell $($ ncor $=2)$. For the right panels of (a) and (b), the two atoms are treated equivalently (i.e., the self-energy is assumed to be identical).

\begin{tabular}{ccccc}
\hline$l$ & $F_{0}$ & $F_{2}$ & $F_{4}$ & $F_{6}$ \\
\hline 1 & $U$ & $5 J$ & - & - \\
2 & $U$ & $\frac{14}{1.0+0.63} J$ & $0.63 F_{2}$ & - \\
3 & $U$ & $\frac{6435}{286+195 \times \frac{451}{675}+250 \times \frac{1001}{2025}} J$ & $\frac{451}{675} F_{2}$ & $\frac{1001}{2025} F_{2}$ \\
\hline
\end{tabular}

Table 2: Formula of the effective Slater integrals for interaction $=$ slater_uj.

and local_potential_factor. local_potential_matrix describes, in the Python dictionary format, a set of inequivalent shell indices $i s h$ and a filename that defines the local potential matrix. The parameter local_potential_factor defines a prefactor to the potential matrix. For details, see DCore's online manual.

2. [system] block

This block includes thermodynamic parameters and some technical parameters such as the inverse temperature and the number of Matsubara frequencies. It is also possible to specify whether the chemical potential is fixed; if it is fixed, its value can be included. There is also the option with_dc, used to consider double-counting correction. When double-counting correction is considered, the following part of the self-energy is subtracted to avoid the double-counting error of the self-energy:

$$
\Sigma_{i, \alpha \sigma \beta \sigma^{\prime}}^{\mathrm{dc}-\mathrm{imp}}=\delta_{\sigma \sigma^{\prime}} \sum_{\gamma \delta \sigma_{1}} U_{\alpha \gamma \beta \delta}\left\langle c_{\gamma \sigma_{1}}^{\dagger} c_{\delta \sigma_{1}}\right\rangle_{0}-\sum_{\gamma \delta} U_{\alpha \gamma \beta \delta}\left\langle c_{\gamma \sigma^{\prime}}^{\dagger} c_{\delta \sigma}\right\rangle_{0},
$$

where $\langle\cdots\rangle_{0}$ indicates the expectation value at the initial (Kohn-Sham) state.

3. [impurity_solver] block

This block specifies the impurity solver to be used and the necessary parameters for 
running the solver program. In addition, the solver-dependent parameters can be specified in this block. For details, see "Impurity solvers" in "Reference Manual" in DCore's online manual.

4. [control] block

This block includes parameters that control the self-consistency loop of DMFT. For example, calculation condition parameters, such as the maximum steps of DMFT loops and the convergence criteria, the input file names of the initial self-energies, and some calculation options, such as the restart option, are specified.

5. [tool] block

This block sets the parameters for the Padé approximation used in dcore_post for analytic continuation. In addition, the information of the momentum path to be specified when calculating the momentum-resolved spectrum function is set.

6. [mpi] block

This block includes parameters that are read by dcore and dcore_post. The usage of the [mpi] block is explained in Sec. 6.2.

\subsection{Output-file format}

Output files are generated by each program. The output files for each program are described below.

1. dcore_pre

dcore_pre generates seedname.h5, where seedname is defined in a model block in the input file. It has two groups, namely dft_input and Dcore. See the DFTTools website [4] for details on the data structure in the dft_input group. In the Dcore group, the values of interaction matrix $U_{\alpha \beta \gamma \delta}^{i}$, where $i$ corresponds to the kind of correlated shell and $\alpha, \beta, \gamma, \delta$ denote the spin-orbital indices at each correlated shell, and local potential $V_{s, o 1, o 2}^{i}$, where $s$ denotes the spin and $o 1, o 2$ denote orbitals, are output.

2. dcore

dcore_pre generates seedname.out.h5. All data are stored in the dmft_out group. In this group, the input parameters, the total number of iteration steps, and the physical quantities, such as the local self-energy in the imaginary frequency domain and the chemical potential, at each iteration step are output. The data structure of the self-energy is described in the online document https://issp-center-dev.github.io/DCore/ develop/reference/gf_format.html. For instance, the self-energy at the first iteration for the 0-th inequivalent shell will be stored below /dmft_out/Sigma_iw/ite1/sh0 in seedname. out.h5. An external impurity solver is invoked as a subprocess from the main process of dcore. For each iteration and each inequivalent shell, dcore generates temporary input and output files in the working directory, e.g., work/imp_shello_ite1 for the first iteration for the 0 -th inequivalent shell. The user can check these temporary files to diagnose problems.

3. dcore_check

dcore_check generates three text files, namely iter_mu.dat, iter_sigma.dat, and sigma.dat, and the corresponding figures in the check directory. In iter_mu.dat 
(iter_sigma.dat), the chemical potential (the average self-energy) as a function of the iteration number is output, and in iter_mu.png (iter_sigma-isho.png), the chemical (renormalization factor) potential and its difference from the previous step at each iteration step are plotted. In sigma.dat, the local self-energy at the final step is output, and in sigma_ave.png, the average self-energy of the last seven iterations is plotted. Here, the average is taken as follows:

$$
\Sigma_{\text {Ave }}\left(i \omega_{n}\right)=\frac{\sum_{i}^{\text {shell }} \sum_{\alpha, \beta}^{N_{\text {orb }}^{i}} \Sigma_{\alpha \beta}\left(i \omega_{n}\right)}{\sum_{i}^{\text {shell }} N_{\text {orb }}^{i}} .
$$

The maximum frequency is specified with the parameter omega_check in the [tool] block.

4. dcore_post

dcore_post generates three text files, namely seedname_dos.dat, which includes the density of states, seedname_akw.dat, which includes the single-particle excitation spectrum $A(k, w)$, and

seedname_momdist.dat, which includes the momentum distribution function. A script (seedname_akw.gp) for displaying $A(k, w)$ is also generated at the same time. All files and figures are output in the post directory.

\section{Installation}

DCore is a collection of pure Python programs built on TRIQS 15] and TRIQS/DFTTools [4]. DCore supports TRIQS 1.4.x, 2.1.x, and 2.2.x. Once these prerequisites are installed, the installation of DCore is quick and efficient. The detailed installation instructions can be found at https://issp-center-dev.github.io/DCore/master/install.html. In addition, at runtime, one of the external impurity solvers shown below must be available.

- ALPS/CT-HYB [6]

- ALPS/CT-HYB-SEGMENT 7

- TRIQS/cthyb [3]

- TRIQS/Hubbard-I 12

- pomerol 13 (as Hubbard-I solver and ED solver)

We provide an up-to-date list of supported impurity solvers and short instructions on installation and usage at https://issp-center-dev.github.io/DCore/master/impuritysolvers. html.

\section{$6 \quad$ Examples}

The examples below show how to define a model, how to compute a self-consistent solution, and how to compute physical quantities. 


\subsection{First example: Square-lattice Hubbard model}

As the first example, we consider a single-band Hubbard model on a square lattice. Without the shell index, the Hamiltonian in Eq. (1) is reduced to

$$
\mathcal{H}=\sum_{\boldsymbol{k} \sigma} \epsilon_{\boldsymbol{k}} c_{\boldsymbol{k} \sigma}^{\dagger} c_{\boldsymbol{k} \sigma}+U \sum_{i} n_{i \uparrow} n_{i \downarrow}
$$

where $n_{i \sigma}=c_{i \sigma}^{\dagger} c_{i \sigma}$ is the local number operator. The energy dispersion $\epsilon_{\boldsymbol{k}}$ is given by

$$
\epsilon_{\boldsymbol{k}}=2 t\left(\cos k_{x}+\cos k_{y}\right)+4 t^{\prime} \sin k_{x} \sin k_{y} .
$$

Note that the nearest-neighbor hopping parameter $t$ should be negative to minimize $\epsilon_{\boldsymbol{k}}$ at the $\Gamma$ point. This is an effective model of cuprate superconductors and is one of the most studied models in strongly correlated electron systems.

\subsubsection{Model construction}

We first set up the model using dcore_pre. Parameters for defining a model are provided in the [model] block of the input file, say, square.ini. The square-lattice Hubbard model can be constructed as shown below.

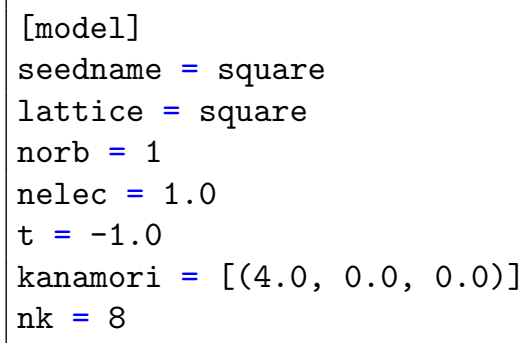

The square lattice is predefined in DCore and can be invoked simply by setting lattice $=$ square. For more complicated models that are not predefined in DCore, this parameter is replaced with lattice $=$ wannier90 and the lattice data are described in a separate file with the Wannier90 format. Sec. 6.1.5 describes its details. We consider half-filling, which is specified by nelec $=1.0$. The value of transfer integral $t$ is specified by $t$. In this case, $t$ is set as -1 , and the absolute value of $t$ is taken as the unit of energy. The interaction parameter $U$ is input by the first component of kanamori. The second and third components correspond to $U^{\prime}$ and $J$, respectively, but are meaningless for single-band models. The system size is determined by $\mathrm{nk}=8$, which means that the $\boldsymbol{k}$-average in Eq. (4) is evaluated by summing up $8 \times 8 \boldsymbol{k}$-points.

With this input file, dcore_pre is executed as

\$ dcore_pre square.ini

This program generates an HDF5 file named square.h5, which includes all the information on the model Hamiltonian, such as $H(k)$.

\subsubsection{Self-consistent calculations}

We now proceed to the main program dcore. For executing the program, we need three additional blocks in the parameter file square.ini, namely [system], [impurity_solver], and [control]. The example below shows a parameter set for a square lattice: 


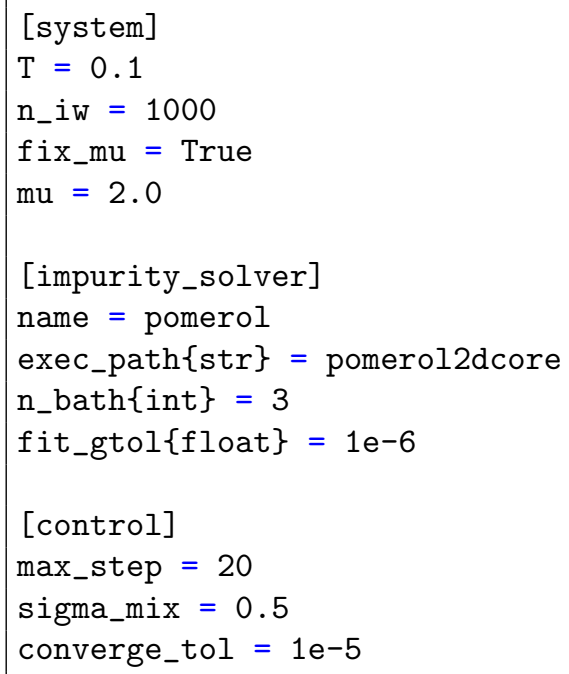

The [system] block includes $\mathrm{T}$ for temperature $T$ and $\mathrm{n}_{-} \mathrm{iw}$ for the number of positive Matsubara frequencies. We fix $\mu$ at 2 by specifying $\mathrm{fix} m u=$ True and $\mathrm{mu}=2.0$ because the condition for half-filling is known. If $f i x \_m u$ is not activated, $\mu$ is adjusted every time the impurity problem is solved to make the occupation number equal to nelec.

The [impurity_solver] block specifies which impurity solver is used and its configuration. In this example, we use the ED solver pomerol, which is specified by name = pomerol. Other parameters in this block are solver-dependent and require the type specification, such as $\{$ str $\}$ and $\{$ int $\}$. The exec_path $\{$ str $\}$ parameter is set to the path to the pomerol2dcore executable. In ED-based solvers, the hybridization function $\Delta_{\sigma}^{\alpha \beta}\left(i \omega_{n}\right)$ in Eq. $(7)$ is approximated by the function composed of $N_{\text {bath }}$ poles:

$$
\tilde{\Delta}_{\sigma}^{\alpha \beta}\left(i \omega_{n}\right)=\sum_{b=1}^{N_{\text {bath }}} \frac{V_{\sigma}^{\alpha b}\left(V_{\sigma}^{\beta b}\right)^{*}}{i \omega_{n}-E_{\sigma}^{b}} .
$$

The energy level $E_{\sigma}^{b}$ and the hybridization parameter $V_{\sigma}^{\alpha b}$ are determined by fitting $\tilde{\Delta}_{\sigma}^{\alpha \beta}\left(i \omega_{n}\right)$ to $\Delta_{\sigma}^{\alpha \beta}\left(i \omega_{n}\right)[1$. The fitting tolerance is specified by fit_gtol. The resultant finite-size system consisting of the correlated site plus $N_{\text {bath }}$ bath sites is solved using numerical diagonalization.

The [control] block includes parameters that control the self-consistent calculations. sigma_mix $=0.5$ means that $\Sigma\left(i \omega_{n}\right)$ are averaged before and after running the solver. The impurity problem is solved max_step $=20$ times at maximum, but the loop is terminated if a convergence criterion is satisfied. The criterion we use is

$$
|O[i]-O[i-1]|<\text { converge_tol, }
$$

where $O[i]$ denotes a quantity at the $i$-th iteration. The quantities $O$ include the chemical potential $\mu$ and the renormalization factor $z$ (see below).

The main program dcore is executed as

\$ dcore --np 4 square.ini

Here, the number of CPU nodes is given after the --np option. The $\boldsymbol{k}$-average of $G\left(\boldsymbol{k}, i \omega_{n}\right)$ in Eq. (4) and the impurity solver are executed in parallel. The results are stored in an HDF5 file named square.out.h5. 

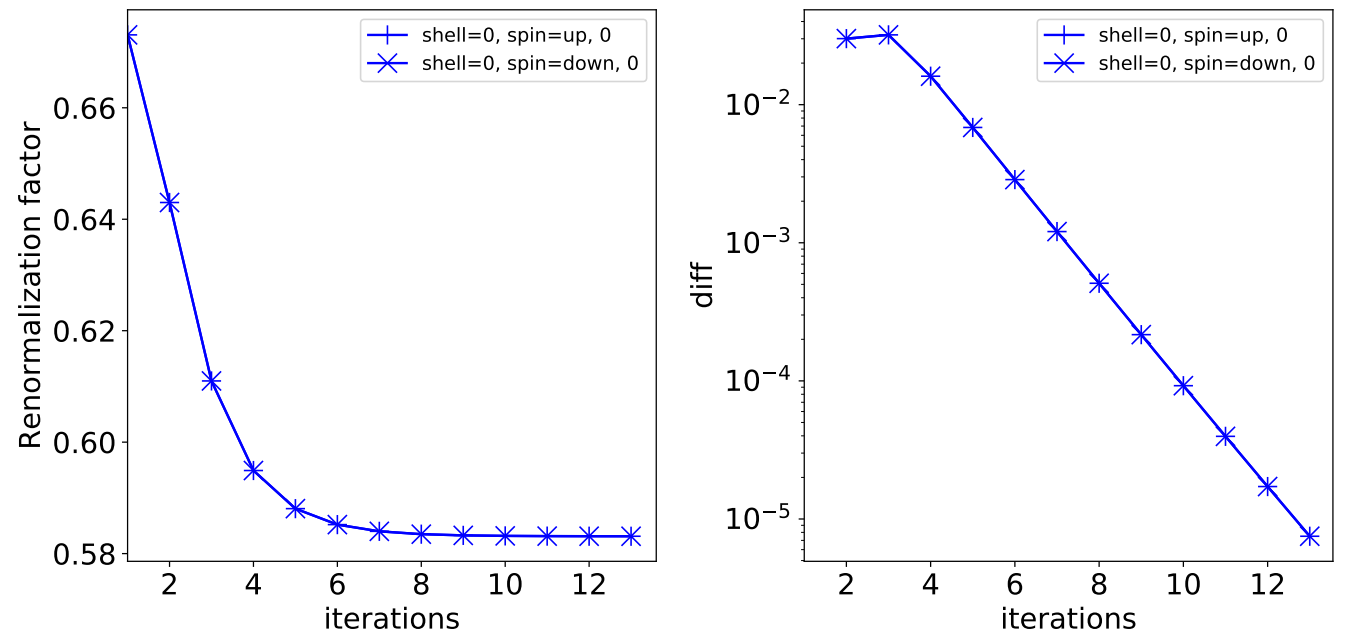

Figure 5: One of the figures generated by dcore_check for a convergence check. The renormalization factor $z$ is plotted as a function of the iteration number (top panel) and its absolute difference between iterations $i$ and $i-1$ is plotted in a logarithmic scale (bottom panel).

After dcore is executed, one must check the convergence of the self-consistent calculations. For this purpose, the auxiliary program dcore_check is provided. dcore_check is called without the --np option as

\$ dcore_check square.ini

Figure 5 shows one of the figures generated by dcore_check. The renormalization factor $z$ defined by

$$
z_{\sigma}=\left[1-\operatorname{Im} \Sigma_{\sigma}\left(i \omega_{0}\right) / \omega_{0}\right]^{-1}
$$

is plotted as a function of the iteration number (top panel). $z$ is bounded in the range $0<z \leq 1$. The bottom panel shows the difference between the $i$-th and $(i+1)$-th iterations. The graph shows an exponential decay. The loop is terminated at the 13 th iteration, where the difference falls below converge_tol $=1 \mathrm{e}-5$.

If the loop does not converge within max_step, one can continue the self-consistent calculations. To this end, a line is added in square.ini,

\section{[control]}

restart $=$ True,

which activates the restart option and executes dcore. sigmamix may be reduced if the convergence graph exhibits oscillation. This is repeated until convergence is reached.

At this point, the DMFT solutions have been obtained for static quantities and $G\left(\boldsymbol{k}, i \omega_{n}\right)$ in the Matsubara domain. For example, the magnetization and the renormalization factor $z$ can be discussed. Figure 6 shows the convergence of $z$ versus the number of bath sites $N_{\text {bath }}$. Convergence to the CT-QMC result (explained in Sec. 6.1.4) is observed for $N_{\text {bath }} \geq 3$. 


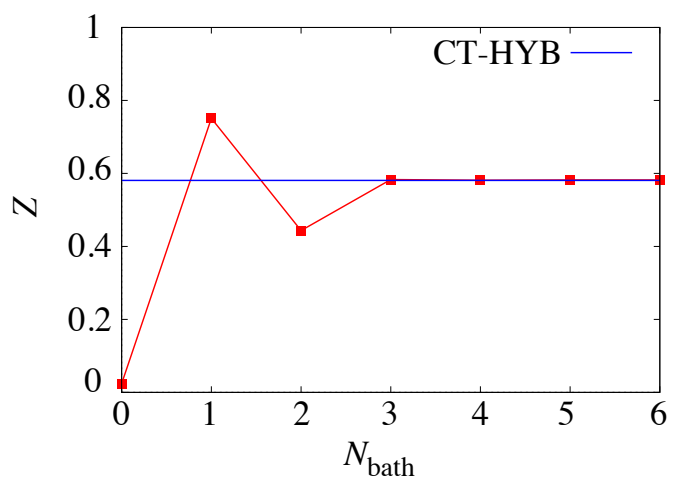

Figure 6: Renormalization factor $z$ as a function of the number of bath sites $N_{\text {bath }}$ computed by the pomerol solver. The blue line shows the CT-QMC result $z=0.58$.

\subsubsection{Dynamical quantities}

After the self-consistent calculations are finished, the post calculation dcore_post is conducted to compute dynamical quantities on the real frequency axis such as the density of states $A(\omega)$ and the single-particle excitation spectrum $A(\boldsymbol{k}, \omega)$. The parameters for this step are provided in the [tool] block as follows:

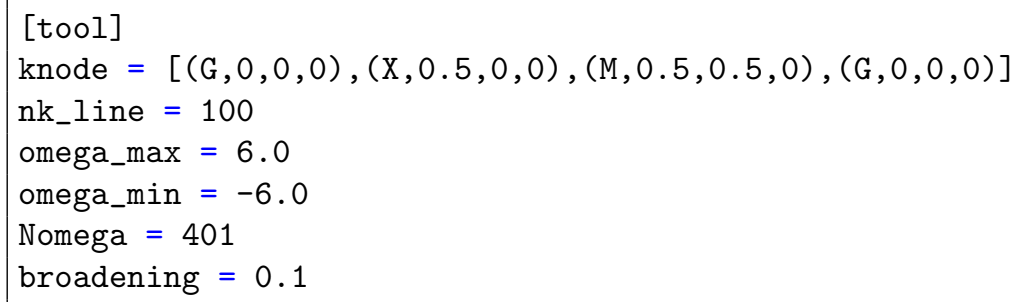

The $\boldsymbol{k}$-path is specified by the parameter knode. In this example, $\boldsymbol{k}$ starts from $\Gamma$ and comes back to $\Gamma$ through $\mathrm{X}$ and $\mathrm{M}$. For each interval, the path is divided into nk_line $=$ 100 points, on which $A(\boldsymbol{k}, \omega)$ is computed. The $\omega$ mesh is generated using the parameters omega_min, omega_max, and Nomega. The parameter broadening specifies the extent of an artificial broadening $\delta$ in the spectrum. This is done by replacing $\omega$ by $\omega+i \delta$ in the analytical continuation. broadening is set to a value on the order of $T$ to obtain a smooth spectrum with the ED solver. For other solvers that treat the thermodynamic limit, one should set broadening $=0$ to avoid artificial broadening.

dcore_post is executed with the --np option because MPI parallel computation is used:

\$ dcore_post --np 4 square.ini

Figure 7(a) shows $A(\boldsymbol{k}, \omega)$ on a given $\boldsymbol{k}$-path. Although the ED solver yielded reasonable estimations for $z$, as shown in Fig. 6, the dynamical quantities clearly show artificial features. Because $\Delta(\omega)$ is composed only of $\mathrm{n}_{-}$bath $=3$ poles located at $\omega=0$ and \pm 1.73 , $A(\boldsymbol{k}, \omega)$ exhibits features of a hybridized band around $\omega= \pm 1.73$. However, the hybridization should take place equally in the whole energy region. A larger $\mathrm{n} \_$bath is necessary to discuss dynamical properties. 

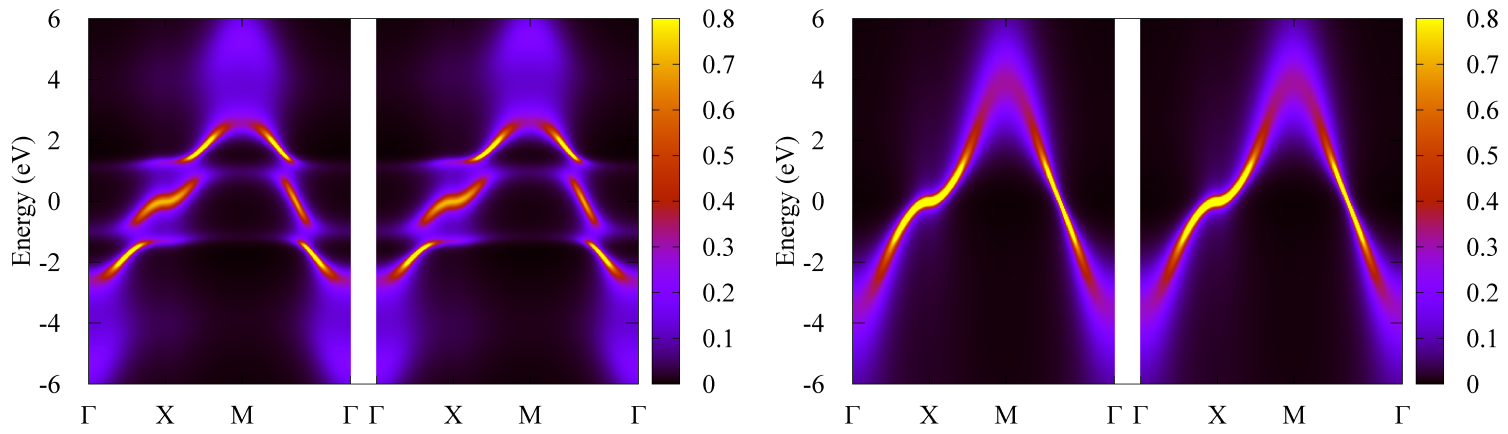

Figure 7: $A(\boldsymbol{k}, \omega)$ computed by (a) the ED solver with three bath sites and (b) the CT-QMC solver.

\subsubsection{CT-QMC solver}

The CT-QMC method offers an unbiased simulation of general interacting models [16, 17]. In particular, efficient calculations for the effective impurity problem are achieved by its hybridization-expansion formulation, which performs Monte Carlo sampling in the expansion with respect to hybridization between the correlated atom and the bath as perturbation [18. For the single-band Hubbard model, an even more efficient algorithm based on a segment picture can be used [19]. Here, we use the ALPSCore [20] version of the implementation developed by Hafermann et al. [21]. Note that only density-density interactions are taken into account in this solver.

To invoke the CT-HYB solver, the parameters in the [impurity_solver] block of square.ini are changed as follows:

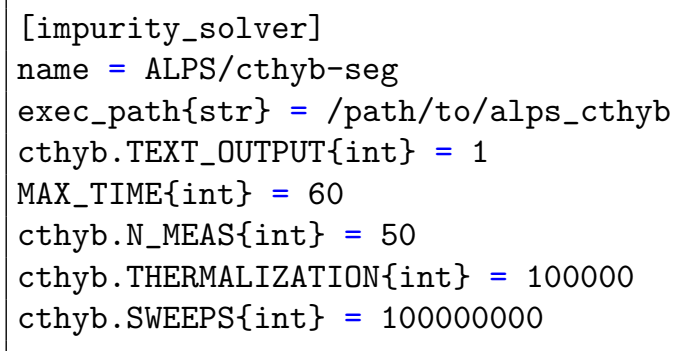

The CT-QMC solver is invoked by name = ALPS/cthyb-seg. The second parameter exec_path $\{$ str $\}$ should be changed to point to the path to the alps_cthyb executable in the environment. Important parameters for the QMC simulation are

- $\operatorname{MAX} \_\operatorname{TIME}\{$ int $\}$,

- cthyb.N_MEAS $\{$ int $\}$,

- cthyb. THERMALIZTION $\{$ int $\}$,

- cthyb.SWEEPS $\{$ int $\}$.

The simulation finishes either when cthyb.SWEEPS\{int $\}$ Monte Carlo sweeps are finished or when the elapsed time reaches MAX_TIME seconds. In this example, cthyb.SWEEPS $\{$ int $\}$ is so 

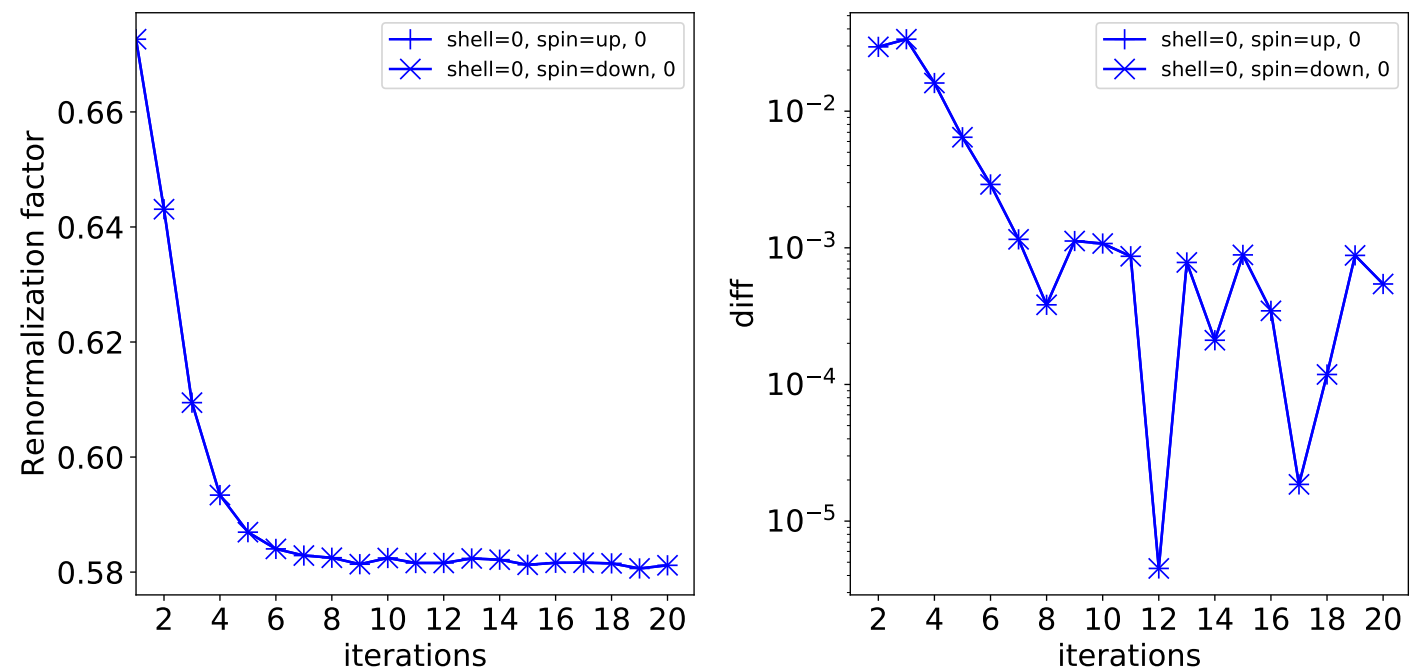

Figure 8: Case of CT-HYB solver for a figure generated by dcore_check for a convergence check. See the caption in Fig. 5.

large that the computation time is controlled by MAX_TIME\{int\}. cthyb. THERMALIZTION int $\}$ specifies the number of updates before the measurement starts. cthyb. THERMALIZTION $\{$ int $\}$ $=100000$ should be large enough to properly discard irrelevant samples. However, this value may not be large enough at lower temperatures. This should be checked by, for example, plotting the histogram of the expansion order [21]. The whole list of optional parameters can be found by running the command

\$ alps_cthyb --help

Note that parameters should be input with the type specification, e.g., $\{$ int $\}$ and $\{$ str $\}$, as in the above example.

Because the QMC solver is time-consuming, it is important to use a proper initial guess for $\Sigma\left(i \omega_{n}\right)$ to reduce the number of iterations. For example, we can use the converged result obtained for similar parameters or the result obtained for the same parameters with a different solver. This can be done by assigning the path of sigma.dat generated by dcore_check to the initial_self_energy parameter as

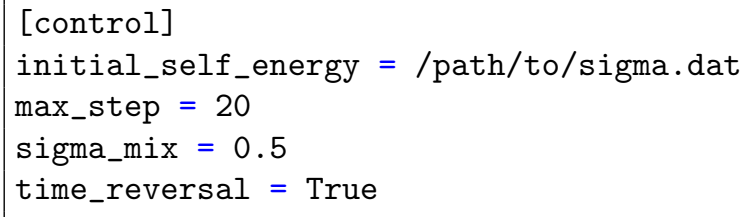

Here, /path/to/sigma. dat should be changed according to the environment. The parameter time_reversal $=$ True activates the spin average of $\Sigma_{\sigma}\left(i \omega_{n}\right)$. This is particularly useful for the QMC solver to improve statistics. Unlike for the ED solver, converge_tol is not specified here, because statistical errors prevent a reliable automatic convergence check. The convergence should instead be checked visually using the graphs generated by dcore_check.

With this input file, all programs, namely dcore_pre, dcore, dcore_check, and dcore_post, are executed as in Sec. 6.1.1. Figure 8 shows a convergence check by dcore_check. The bot- 
tom panel shows that the statistical error is on the order of $10^{-3}$ and that convergence is reached at around the 10th iteration. The result for $A(\boldsymbol{k}, \omega)$ is shown in Fig. 7(b). Note that artificial broadening is turned off by broadening $=0.0$. The spectrum exhibits low-energy quasiparticle excitations and high-energy broadening due to correlations.

\subsubsection{Wannier90 interface}

DCore provides simple lattice models such as a one-dimensional chain, a two-dimensional square lattice, and a three-dimensional cubic lattice. Arbitrary lattices that are not predefined in DCore can be implemented using the Wannier90 format [22]. In the following, we show how to construct the square lattice using Wannier90 input.

The only difference in square.ini is in the [model] block, which includes

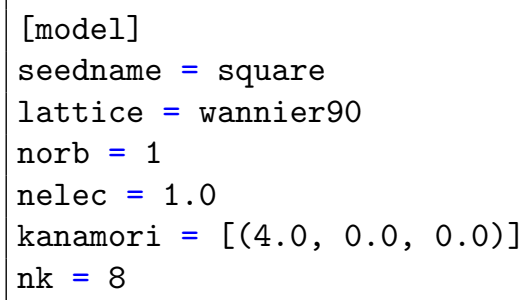

The difference between this block and that in Sec.6.1.1 is lattice $=$ wannier90. When this is given, DCore reads a wannier90 file named seedname_hr. dat (square $\mathrm{hr}$. dat in the present case) to set up the one-body part of the Hamiltonian, $H(\boldsymbol{k})$. square hr. dat describing the square lattice is given by

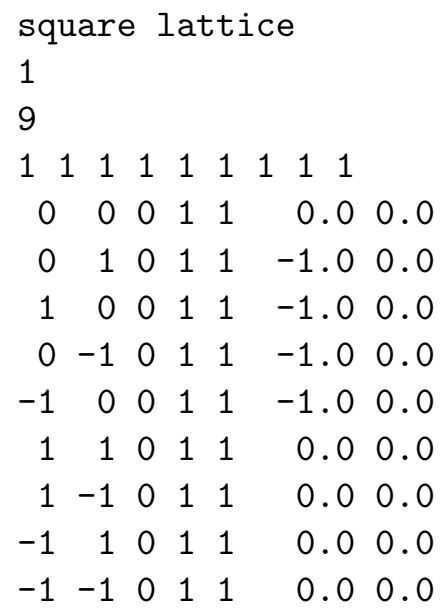

The first line can be any text. The second line specifies the number of orbitals in a unit cell. The next line specifies the number of cells, $N_{\text {cell }}$, for which the transfer integrals are provided. Here, we have $N_{\text {cell }}=9$ cells, as shown in Fig. 9 (a). The value of transfer integrals, $H_{i j}^{\mathrm{W} 90}(\boldsymbol{R})$, in the one-body Hamiltonian $\mathcal{H}=\sum_{\boldsymbol{R}^{\prime} i j} H_{i j}^{\mathrm{W} 90}\left(\boldsymbol{R}^{\prime}-\boldsymbol{R}\right) c_{\boldsymbol{R} i}^{\dagger} c_{\boldsymbol{R}^{\prime} j}$ is listed for all possible combinations of $\boldsymbol{R}, i$, and $j$ for fixed $\boldsymbol{R}^{\prime}=0$. Here, $i$ and $j$ denote the combined orbital and site indices in the unit cell. The format is " $l_{1} l_{2} l_{3} i j \operatorname{Re} H_{i j}^{\mathrm{W} 90}(\boldsymbol{R}) \operatorname{Im} H_{i j}^{\mathrm{W} 90}(\boldsymbol{R})$ ", where $\boldsymbol{R} \equiv l_{1} \boldsymbol{a}_{1}+l_{2} \boldsymbol{a}_{2}+l_{3} \boldsymbol{a}_{3}$ and $\left(\boldsymbol{a}_{1}, \boldsymbol{a}_{2}, \boldsymbol{a}_{3}\right)$ are the unit lattice vectors. The convention used by Wannier90 is related to the one in Eq. $(2)$ as $H_{i j}^{\mathrm{W} 90}(-\boldsymbol{R})=H_{i j}(\boldsymbol{R})$. 
(a)

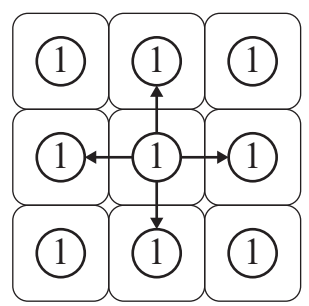

(b)

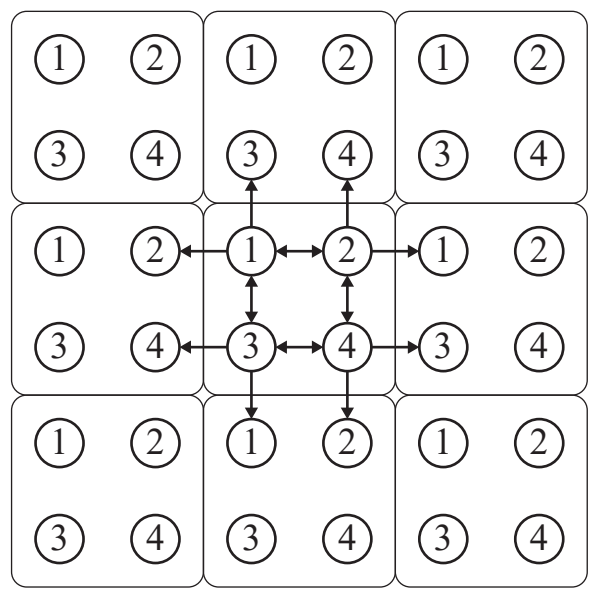

Figure 9: Lattice configuration for the Wannier90 input for (a) the paramagnetic state and (b) the AFM state. The circles show sites (atoms) and the number is the label of the site in the unit cell indicated by the square. The arrows indicate the hopping included in the input file.

\subsubsection{Antiferromagnetic state}

With the Wannier90 format, one can construct arbitrary lattices. For example, we can enlarge the unit cell to allow an antiferromagnetic (AFM) solution. Let us see how this is done for the square lattice example.

Figure 9(b) shows the lattice configuration for AFM calculations. Four "orbitals" are contained in a unit cell. square $\mathrm{hr}$. dat now includes the hopping inside the unit cell ( 8 double-pointed arrows) and the hopping from the central cell to neighboring unit cells (8 single-pointed arrows).

Parameters in the [model] block of square.ini should be modified as follows:

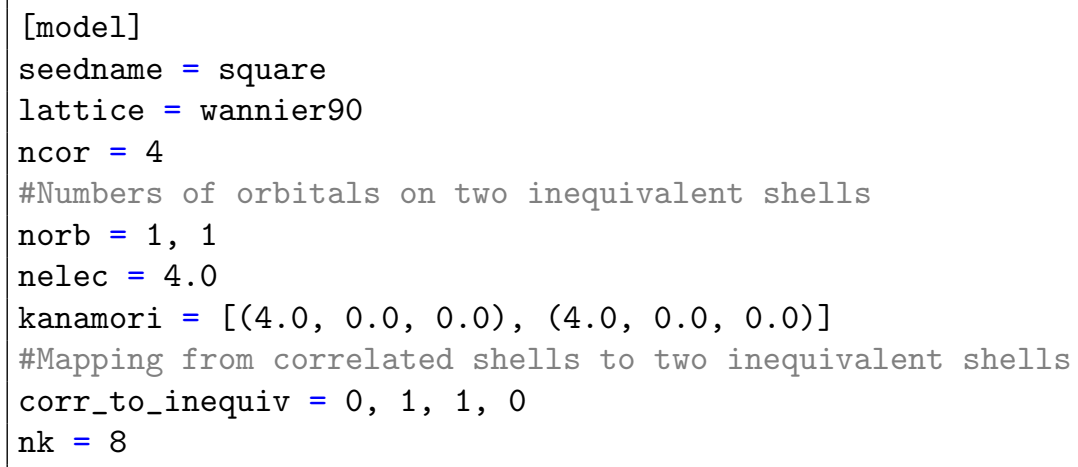

The total number of orbitals contained in the unit cell is specified by ncor $=4$. nelec is the total number of electrons in the unit cell, and hence needs to be multiplied by 4 . In the AFM state, we expect a staggered ordered state, where site 1 and site 4 are equivalent (e.g., spin-up state), and site 2 and site 3 are equivalent (spin-down state). This constraint can be imposed by setting corr_to_inequiv $=0,1,1,0$, which indicates that there are only two 
inequivalent sites: site 1 and site 4 are labeled with 0 , and site 2 and site 3 are labeled with 1 . Two independent impurity problems are solved in each step of the self-consistent calculations. For each impurity model, the number of orbitals and the strength of interactions are specified by the norb and kanamori parameters, respectively.

To obtain an AFM solution, we need to start the self-consistent calculation with a brokensymmetry initial guess. To this end, we set the initial self-energy to

$$
\Sigma_{\alpha \beta}^{\sigma}\left(i \omega_{n}, S\right)=v_{\alpha \beta}^{\sigma}(S)
$$

using local potential $v_{\alpha \beta}^{\sigma}(S)$, which depends on the inequivalent site $S$. The [control] block in square.ini contains an additional parameter that specifies the initial self-energy as

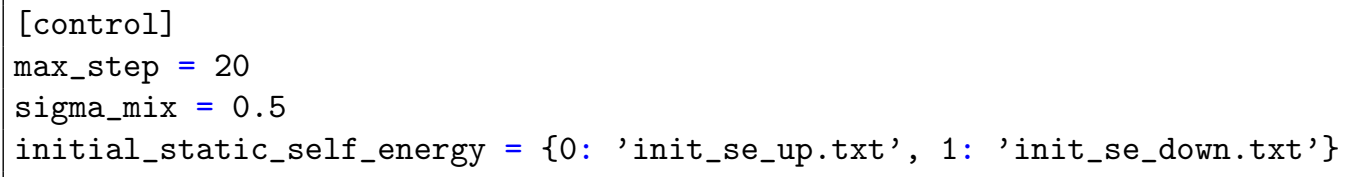

A path to a text file is given in initial_static_self_energy for each inequivalent site using the dictionary literal in Python. The text files describe non-zero components in $v_{\alpha \beta}^{\sigma}$ in the form " $\sigma \alpha \beta \operatorname{Re} v_{\alpha \beta}^{\sigma} \operatorname{Im} v_{\alpha \beta}^{\sigma}$ ". To obtain a magnetic state (spin-up state), we can input the magnetic field in init_se_up.txt as

$\begin{array}{llllllll}0 & 0 & 0 & -1.0 & 0.0\end{array}$

10001.00 .0

and the magnetic field with the opposite direction is given in init_se_down.txt as

00001.00 .0

$\begin{array}{lllllll}1 & 0 & 0 & -1.0 & 0.0\end{array}$

The parameters in [system] and [impurity_solver] do not change from the paramagnetic calculations.

Figure 10 shows the convergence of the spin moment $m_{\xi}(S)(\xi=x, y, z)$ for inequivalent site $S=0$. A convergence of $m_{z}$ to a non-zero value was obtained around the 30 th iteration. The moment $m_{z}=0.283$ is about half the full moment $(1 / 2)$. We confirmed that $m_{z}(S)$ for $S=1$ converges to the negative side, namely $m_{z}(0)=-m_{z}(1)$ (no figure). The parameters $U=4, n=1$, and $T=0.1$ are the same as those in the previous calculations in Fig. 7 , meaning that the paramagnetic solution was not a true solution of the DMFT equation. The phase diagram is shown, for example, in Ref. 23].

\subsection{Solving multi-orbital model using QMC solver: $t_{2 \mathrm{~g}}$ model on a Bethe lattice}

In this subsection, we show how to solve a three-orbital model on a Bethe lattice by using DCore. In this model, an interesting phenomenon called spin-freezing transition occurs [24]. Spin-freezing is signaled by a peculiar frequency dependence of the self-energy: $\operatorname{Im} \Sigma\left(i \omega_{n}\right) \propto$ $\omega_{n}^{0.5}$. We will reproduce this result using DCore. 

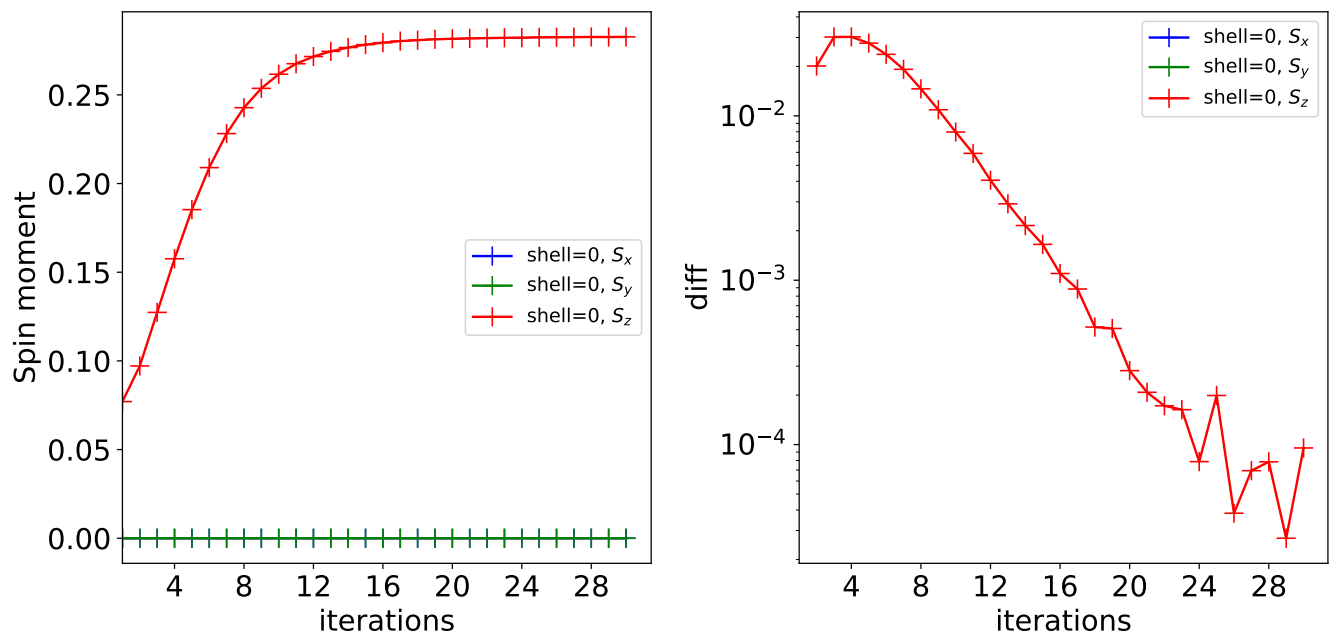

Figure 10: Spin moment $m_{\xi}(S)(\xi=x, y, z)$ for inequivalent site $S=0$ as a function of the iteration number. The top panel shows $m_{\xi}(S)$ itself and the bottom panel shows the difference between iterations $i$ and $i-1$.

\subsubsection{Model definition}

We first make the input file of dcore_pre for generating an HDF5 file that is necessary for DMFT calculations. The Hamiltonian in Ref. [24] is defined as

$$
\begin{aligned}
\mathcal{H} & =\sum_{k, \sigma} \epsilon_{k}^{\alpha} d_{k, \sigma}^{\alpha \dagger} d_{k, \sigma}^{\alpha}+\sum_{i} \mathcal{H}_{\mathrm{loc}}^{i}, \\
\mathcal{H}_{\mathrm{loc}}^{i} & =-\sum_{\alpha, \sigma} \mu n_{\alpha, \sigma}+\sum_{\alpha} U n_{\alpha \uparrow} n_{\alpha \downarrow}+\sum_{\alpha>\beta, \sigma} U^{\prime} n_{\alpha, \sigma} n_{\beta,-\sigma}+\left(U^{\prime}-J\right) n_{\alpha, \sigma} n_{\beta, \sigma} \\
& -\sum_{\alpha \neq \beta} J\left(c_{\alpha \downarrow}^{\dagger} c_{\beta \uparrow}^{\dagger} c_{\beta \downarrow} c_{\alpha \uparrow}+c_{\beta \uparrow}^{\dagger} c_{\beta \downarrow}^{\dagger} c_{\alpha \uparrow} c_{\alpha \downarrow}\right),
\end{aligned}
$$

where $\alpha=1,2,3$ is the orbital index. We use a Kanamori interaction with $U=8, U^{\prime}=$ $U-2 J=5.3333333$, and $J=1.33333$. We use the same model parameters as those used in the paper [24]: $n=1.6, t=1.0, U / t=8.0, J / U=1 / 6$. The input file for dcore_pre is as follows:

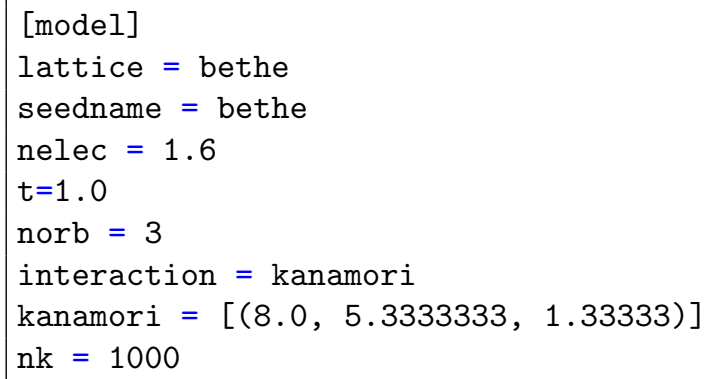

After running dcore_pre, an HDF5 file named bethe.h5 is generated. DCore discretizes the density of states of the Bethe lattice, a semicircular density of states on $[-2 t, 2 t]$, along a virtual one-dimensional $k$ axis with $\mathrm{nk}=1000$ points. 


\subsubsection{DMFT calculation}

Next, we make the input file of dcore. The calculation in Ref. 24] was done using the matrix formalism of the CT-HYB method. To make a direct comparison, we use an implementation of the same algorithm, ALPS/CT-HYB, which was developed by one of the authors. An example input file of dcore is shown below.

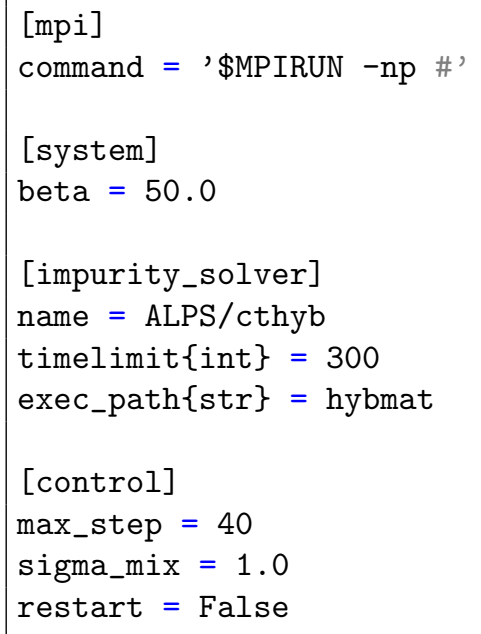

In this example file, we omit the [model] block, which is the same as that in the input file of dcore_pre. The inverse temperature $\beta$ is set to $50 t$. We also define a command for MPI parallelization using the [mpi] block. Here, \# in command of the mpi block is replaced by the number of processes specified at runtime. In the above example, we define the environment variable MPIRUN and run the program with 24 MPI processes as follows.

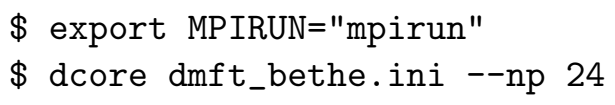

After running dcore, the results of the self-energy and Green's functions in each iteration are accumulated in an HDF5 file named *seedname*.out.h5 (bethe. out.h5 in the present case) and a text file that contains the self-energies (sigma.dat) is output in the check directory.

Before analyzing the computed results such as the self-energy, the user may check the convergence of CT-QMC sampling. The impurity solver runs for 300 seconds at each iteration step, and the standard output and error of the solver are redirected to the standard output of dcore. The last part of the output of the solver at the last iteration looks as follows. The perturbation orders measured just before and after the measurement steps are close to each other $(\simeq 58)$, indicating that the thermalization time was long enough: ALPS/CT-HYB used $10 \%$ of the total simulation time (timelimit) for thermalization. One can see that the average sign is close to 1 , indicating that there is no severe sign problem.

$====$ Thermalization analysis ====

Perturbation orders just before and after measurement steps are 57.6833 and 57.9479.

$====$ Number of Monte Carlo steps spent in configuration spaces ====

$\mathrm{Z}$ function: 5.03099

G1: 4.97089 


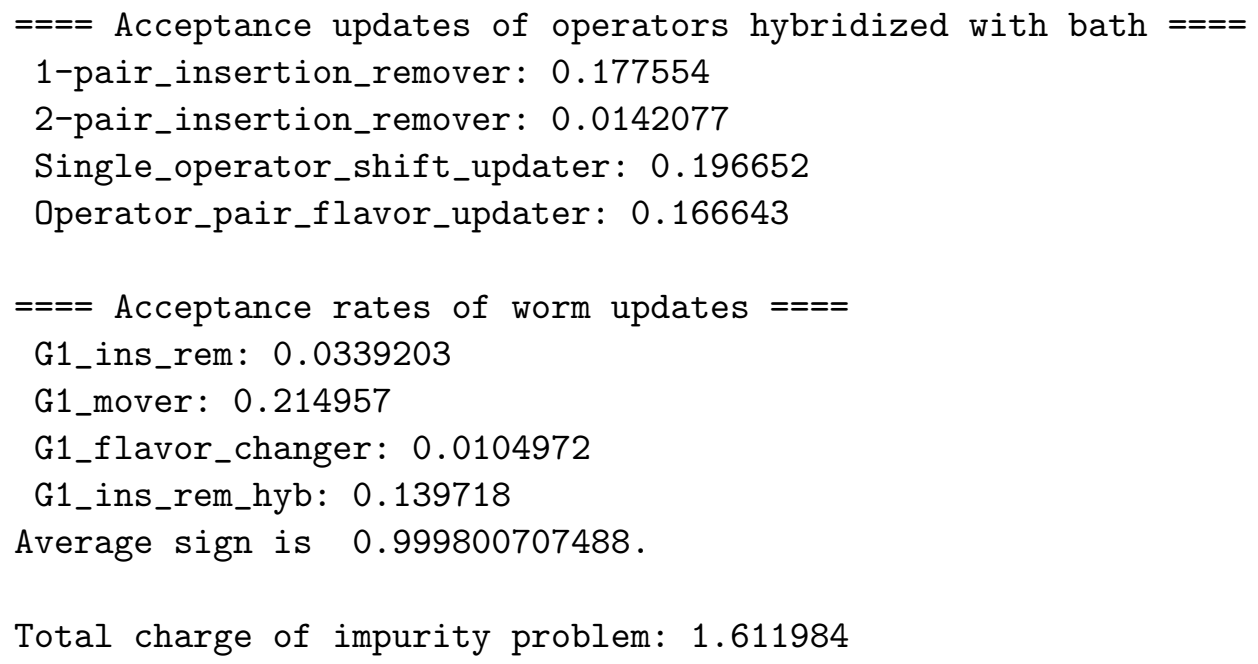

After checking the convergence of the self-consistent iterations using dcore_check, one can plot the self-energy stored in sigma.dat as shown in Fig. 11. The solid line shows the results taken from 24. In the low-frequency region, $\operatorname{Im} \Sigma\left(i \omega_{n}\right)$ is proportional to $\omega_{n}^{1 / 2}$. The three symbols represent the orbital diagonal components of $\operatorname{Im} \Sigma\left(i \omega_{n}\right)$ computed by DCore. The results obtained by dcore match those of [24].

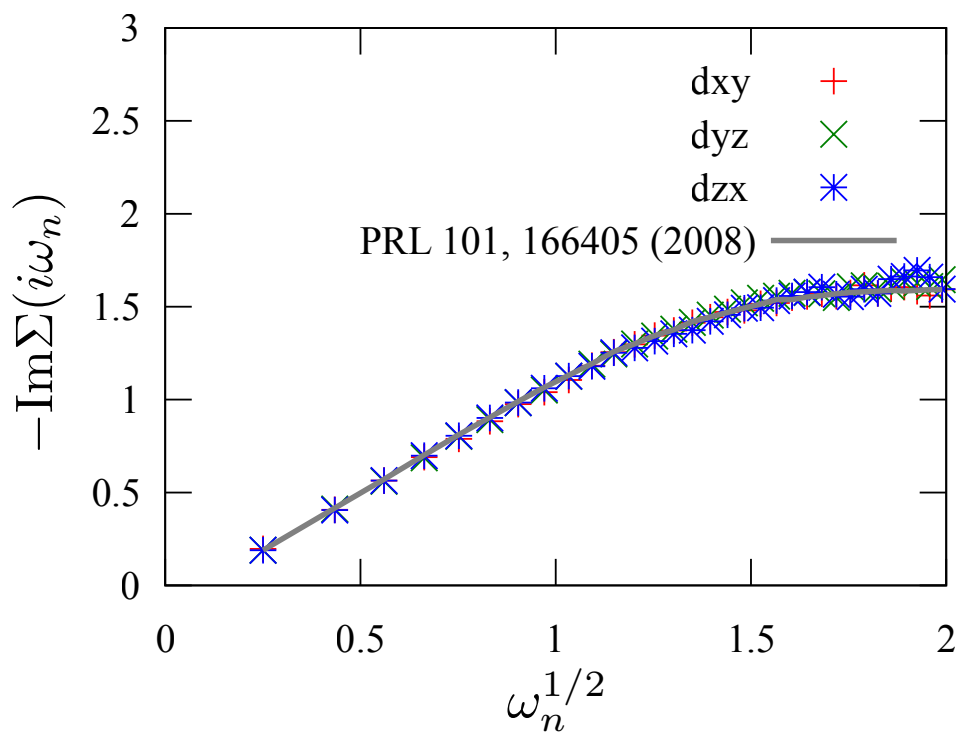

Figure 11: Matsubara frequency dependence of $\operatorname{Im} \Sigma\left(i \omega_{n}\right)$. The solid line indicates the numerical results reported in Ref. 24]. The three symbols indicate $\operatorname{Im} \Sigma\left(i \omega_{n}\right)$ of $d_{x y}, d_{y z}$, and $d_{z x}$ orbitals, respectively, obtained by DCore.

\subsection{DFT+DMFT exmaple: $\mathrm{SrVO}_{3}$}

In this section, we demonstrate how to perform a multi-orbital DFT+DMFT calculation for a realistic band structure. In particular, we use $\mathrm{SVO}_{3}$ which has been studied by DMFT as 
a test bed in many previous studies.

\subsubsection{Construction of Wannier functions}

We fist construct maximally localized Wannier functions for the $t_{2 \mathrm{~g}}$ manifold using Quantum ESPRESSO [25] and Wannier90 [22]. Any DFT programs that support Wannier90 may be used alternatively. For our DFT calculation, we take a lattice constant of 7.29738 a.u and construct a three-orbital tight-binding model following a common procedure using the local density approximation (LDA). We provide more detailed description on how to construct Wannier functions using Quantum ESPRESSO or another DFT program, OpenMX [26] at https: //issp-center-dev.github.io/DCore/master/tutorial/srvo3/srvo3.html. There, we can download input files as well as the data file of the resultant tight-binding model.

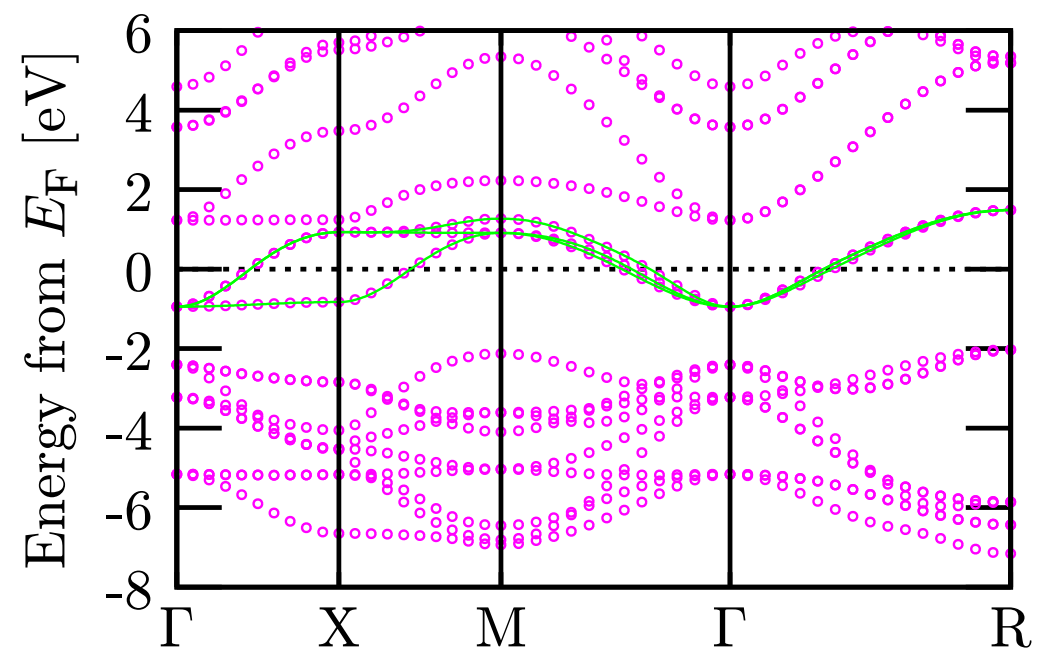

Figure 12: Comparison of LDA band structure and fit by the Wannier functions. The circles denote the energies computed by LDA and the solid lines denote the Wannier fit.

\subsubsection{Model definition}

We show the model block of our input file for performing a DMFT calculation using the Wannier functions below. We adopted the Kanamori interaction parameters $(U=3.419 \mathrm{eV}$, $U^{\prime}=2.315 \mathrm{eV}, J=0.530 \mathrm{eV}$ ) estimated in Ref. [27]. The number of grid points in the first Brillouin zone along each reciprocal vector is set to 10 . This number does not have to match the one used in the DFT calculation. The total number of $k$ points is $10^{3}$.

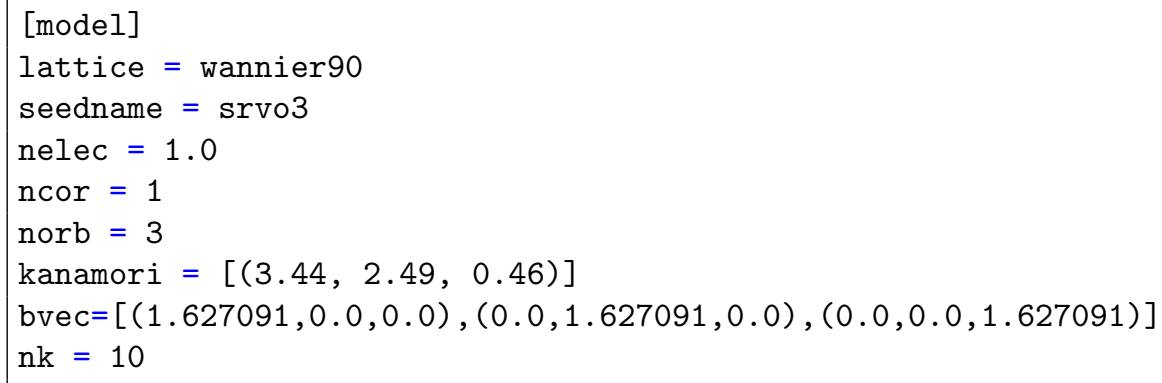




\subsubsection{Parameters for self-consistent calculations and post processing}

We set input parameters for ALPS/CT-HYB in the impurity_solver block. The parameters timelimit and exec_path are passed to ALPS/CT-HYB. The impurity solver runs for 100 seconds with $96 \mathrm{MPI}$ processes at each self-consistent iteration. We set an initial guess for the chemical potential to an appropriate value and inverse temperature to $\beta=20 \mathrm{eV}^{-1} \simeq 580 \mathrm{~K}$.

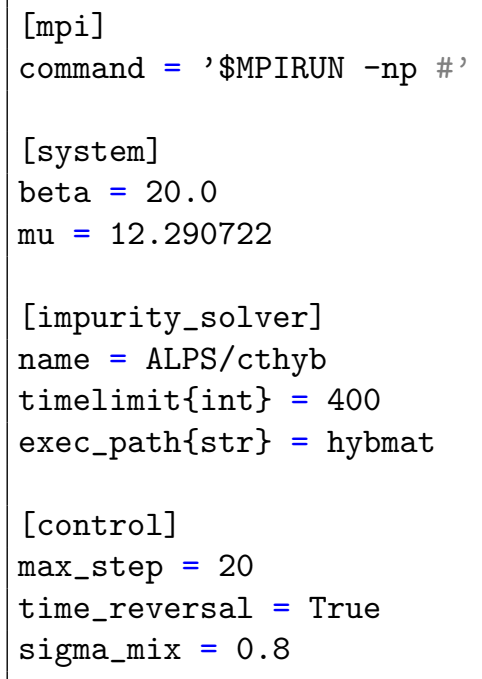

The tool block includes input parameters for post processing. In this example, we compute $A(k, \omega)$ using dcore_post along the $k$-paths specified by symmetry points given in knode. As set by the parameter omega_pade, the data of the self-energy in the energy window of $0<\omega_{n} \leq 2$ is used for analytic continuation to the real-frequency axis. Here, we introduce a small broadening factor for computing spectral functions.

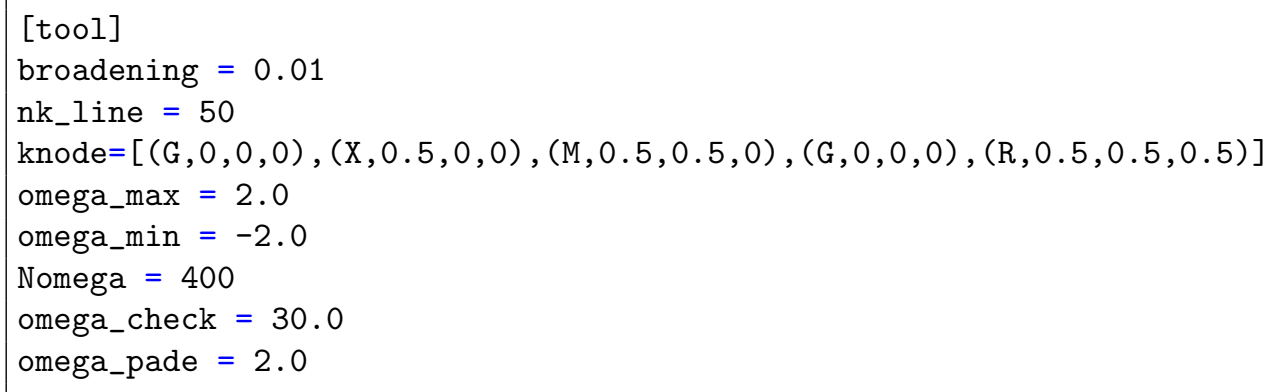

\subsubsection{Results}

In Fig. 12, one can see that the $t_{2 \mathrm{~g}}$ bands are fitted very well by the Wannier functions. The $t_{2 \mathrm{~g}}$ manifold is separated in energy space from the $e_{\mathrm{g}}$ bands above $1.5 \mathrm{eV}$ and oxygen bands below $-2 \mathrm{eV}$. Since we constructed the $t_{2 \mathrm{~g}}$ model, the $e_{\mathrm{g}}$ bands and oxygen bands are not taken into account in the present DMFT calculation. Figure 13 shows $A(\boldsymbol{k}, \omega)$ computed by DMFT Compared to the DFT band structure in Fig. 12, one can see that the $t_{2 \mathrm{~g}}$ band is substantially renormalized by strong correlation effects as expected.

There are several previous DMFT studies for this compounds 28 30]. These calculations aimed at a quantitative description of the spectrum of the real material and therefore took into account other bands such as the $e_{\mathrm{g}}$ bands and oxygen bands. Thus, a direct comparison with our result is not possible. 


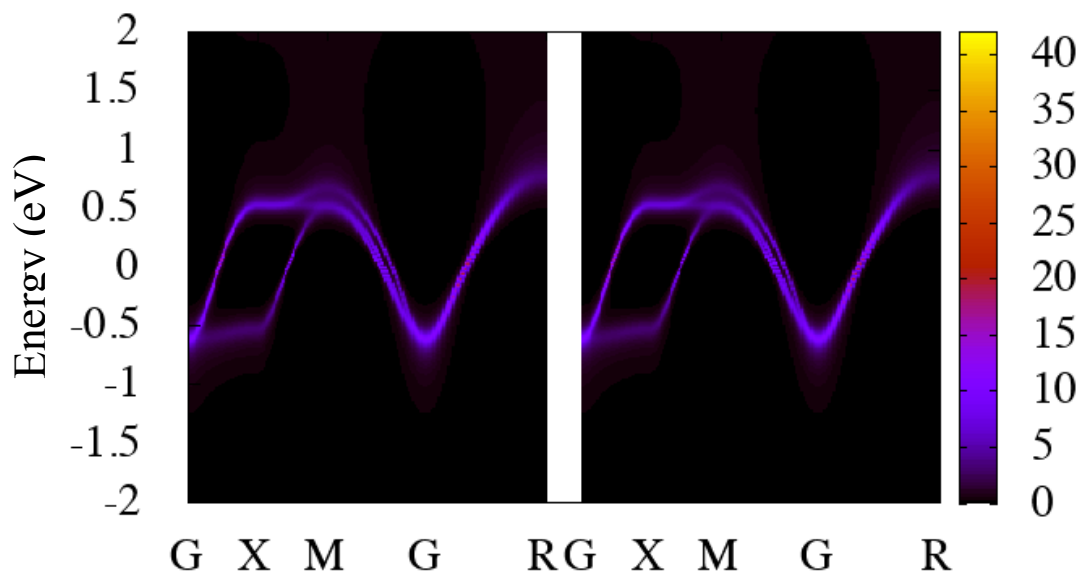

Figure 13: $A(\boldsymbol{k}, \omega)$ computed by DMFT for the Wannier model of $\mathrm{SrVO}_{3}$.

For DCore, one could take into account those uncorrelated bands by constructing Wannier functions for the full $3 d$ orbitals and oxygen $p$ orbitals in a wider energy window. In this case, we may have to carefully adjust (manually) the level splitting between $t_{2 \mathrm{~g}}$ and the uncorrelated orbitals because the current version of DCore does not support intra-shell (site) interactions between the $d$ and $p$ shells.

\section{Conclusion}

We introduced the open-source software package DCore, which is integrated DMFT software for correlated electrons. We described the algorithm, the structure of the package, installation, and file format, and demonstrated usage for the single-/multi-orbital Hubbard models and the Wannier90 model for $\mathrm{SrVO}_{3}$.

We are planning to implement more functionality in a future version. The current version supports only the Padé approximation for analytic continuation. However, the Padé approximation is sensitive to statistical noise. A future version will support external analytic continuation solvers based on more stable methods such as SpM 31 and Maxent 32. Furthermore, we will implement recently proposed efficient methods for computing static/dynamical lattice susceptibilities based on the Bethe-Salpeter equation [23, 33]. DCore will support Python 3 as soon as TRIQS is upgraded to Python 3. There are also plans to support more external impurity solvers such as iQIST [34] and w2dynamics [9].

\section{Acknowledgements}

We acknowledge Takeo Kato and Yuichi Motoyama for supporting the DCore project. We acknowledge the TRIQS community and the ALPS community for developing useful opensource libraries. 
Author contributions For an updated record of individual contributions, consult the repository at https://issp-center-dev.github.io/DCore/master/index.html.

Funding information H.S., J.O., and K.Y. were supported by JSPS KAKENHI Grant No. 18H01158. H.S. was supported by JSPS KAKENHI Grant No. 16K17735. J.O. was supported by JSPS KAKENHI Grant No. 18H04301 (J-Physics). N.T. was supported by JSPS KAKENHI Grant No. 19H05817, and 19H05820. K.Y. was supported by JSPS KAKENHI Grant No. 19K03649, and Building of Consortia for the Development of Human Resources in Science and Technology, MEXT, Japan.

\section{References}

[1] A. Georges, G. Kotliar, W. Krauth and M. J. Rozenberg, Dynamical mean-field theory of strongly correlated fermion systems and the limit of infinite dimensions, Reviews of Modern Physics 68(1), 13 (1996).

[2] G. Kotliar, S. Y. Savrasov, K. Haule, V. Oudovenko, O. Parcollet and C. Marianetti, Electronic structure calculations with dynamical mean-field theory, Reviews of Modern Physics 78(3), 865 (2006), doi:10.1103/revmodphys.78.865.

[3] P. Seth, I. Krivenko, M. Ferrero and O. Parcollet, TRIQS/CTHYB: A continuous-time quantum Monte Carlo hybridisation expansion solver for quantum impurity problems, Computer Physics Communications 200, 274 (2016).

[4] M. Aichhorn, L. Pourovskii, P. Seth, V. Vildosola, M. Zingl, O. E. Peil, X. Deng, J. Mravlje, G. J. Kraberger, C. Martins, M. Ferrero and O. Parcollet, TRIQS/DFTTools: A TRIQS application for ab initio calculations of correlated materials, Computer Physics Communications 204, 200 (2016).

[5] B. Bauer, L. D. Carr, H. G. Evertz, A. Feiguin, J. Freire, S. Fuchs, L. Gamper, J. Gukelberger, E. Gull and S. Guertler, The ALPS project release 2.0: open source software for strongly correlated systems, Journal of Statistical Mechanics: Theory and Experiment 2011(05), P05001 (2011), doi:10.1088/1742-5468/2011/05/p05001.

[6] H. Shinaoka, E. Gull and P. Werner, Continuous-time hybridization expansion quantum impurity solver for multi-orbital systems with complex hybridizations, Computer Physics Communications 215, 128 (2017).

[7] https://github.com/ALPSCore/CT-HYB-SEGMENT.

[8] H. Shinaoka, Y. Nomura and E. Gull, Efficient implementation of the continuous-time interaction-expansion quantum Monte Carlo method, arXiv (2018), 1807.05238.

[9] M. Wallerberger, A. Hausoel, P. Gunacker, A. Kowalski, N. Parragh, F. Goth, K. Held and G. Sangiovanni, w2dynamics: Local one- and two-particle quantities from dynamical mean field theory, Computer Physics Communications 235, 388 (2018), doi:10.1016/j.cpc.2018.09.007, 1801.10209 
[10] V. Singh, U. Herath, B. Wah, X. Liao, A. H. Romero and H. Park, DMFTwDFT: An open-source code combining Dynamical Mean Field Theory with various Density Functional Theory packages, arXiv (2020), 2002.00068.

[11] http://hauleweb.rutgers.edu/tutorials/index.html.

[12] https://triqs.github.io/hubbardI.

[13] A. E. Antipov, I. Krivenko and S. Iskakov, aeantipov/pomerol: 1.2 (2017), doi: $10.5281 /$ zenodo.825870.

[14] M. Kawamura, K. Yoshimi, T. Misawa, Y. Yamaji, S. Todo and N. Kawashima, Quantum lattice model solver hphi, Comput. Phys. Commun. 217, 180 (2017), doi:https://doi.org/10.1016/j.cpc.2017.04.006

[15] O. Parcollet, M. Ferrero, T. Ayral, H. Hafermann, I. Krivenko, L. Messio and P. Seth, TRIQS: A toolbox for research on interacting quantum systems, Computer Physics Communications 196, 398 (2015).

[16] A. Rubtsov, V. Savkin and A. Lichtenstein, Continuous-time quantum Monte Carlo method for fermions, Physical Review B 72(3), 035122 (2005).

[17] E. Gull, A. J. Millis, A. I. Lichtenstein, A. N. Rubtsov, M. Troyer and P. Werner, Continuous-time Monte Carlo methods for quantum impurity models, Reviews of Modern Physics 83(2), 349 (2011).

[18] P. Werner and A. Millis, Hybridization expansion impurity solver: General formulation and application to Kondo lattice and two-orbital models, Physical Review B 74(15), 155107 (2006).

[19] P. Werner, A. Comanac, L. de' Medici, M. Troyer and A. Millis, Continuous-Time Solver for Quantum Impurity Models, Physical Review Letters 97(7), 076405 (2006).

[20] A. Gaenko, A. E. Antipov, G. Carcassi, T. Chen, X. Chen, Q. Dong, L. Gamper, J. Gukelberger, R. Igarashi, S. Iskakov, M. Könz, J. P. F. LeBlanc et al., Updated core libraries of the ALPS project, Computer Physics Communications 213, 235 (2017).

[21] H. Hafermann, P. Werner and E. Gull, Efficient implementation of the continuous-time hybridization expansion quantum impurity solver, Computer Physics Communications 184(4), 1280 (2013).

[22] G. Pizzi, V. Vitale, R. Arita, S. Blgel, F. Freimuth, G. Granton, M. Gibertini, D. Gresch, C. Johnson, T. Koretsune, J. Ibaez-Azpiroz, H. Lee et al., Wannier90 as a community code: new features and applications, Journal of Physics: Condensed Matter 32(16), 165902 (2020), doi:10.1088/1361-648x/ab51ff.

[23] J. Otsuki, K. Yoshimi, H. Shinaoka and Y. Nomura, Strong-coupling formula for momentum-dependent susceptibilities in dynamical mean-field theory, Physical Review B 99(16), 165134 (2019), doi:10.1103/physrevb.99.165134.

[24] P. Werner, E. Gull, M. Troyer and A. J. Millis, Spin freezing transition and nonfermi-liquid self-energy in a three-orbital model, Phys. Rev. Lett. 101, 166405 (2008), doi:10.1103/PhysRevLett.101.166405. 
[25] P. Giannozzi, O. Andreussi, T. Brumme, O. Bunau, M. B. Nardelli, M. Calandra, R. Car, C. Cavazzoni, D. Ceresoli, M. Cococcioni, N. Colonna, I. Carnimeo et al., Advanced capabilities for materials modelling with Quantum ESPRESSO, Journal of Physics: Condensed Matter 29(46), 465901 (2017), doi:10.1088/1361-648x/aa8f79.

[26] T. Ozaki, Variationally optimized atomic orbitals for large-scale electronic structures, Physical Review B 67(15), 155108 (2003), doi:10.1103/physrevb.67.155108.

[27] Y. Nomura, M. Kaltak, K. Nakamura, C. Taranto, S. Sakai, A. Toschi, R. Arita, K. Held, G. Kresse and M. Imada, Effective on-site interaction for dynamical mean-field theory, Physical Review B 86(8), 085117 (2012), doi:10.1103/physrevb.86.085117, 1205.2836.

[28] I. A. Nekrasov, K. Held, G. Keller, D. E. Kondakov, T. Pruschke, M. Kollar, O. K. Andersen, V. I. Anisimov and D. Vollhardt, Momentum-resolved spectral functions of SrVO 3 calculated by LDA + DMFT, Physical Review B 73(15), 155112 (2006), doi:10.1103/physrevb.73.155112, cond-mat/0508313.

[29] R. Sakuma, P. Werner and F. Aryasetiawan, Electronic structure of SrVO3 within $G W+D M F T$, Physical Review B 88(23), 235110 (2013), doi:10.1103/physrevb.88.235110.

[30] C. Taranto, M. Kaltak, N. Parragh, G. Sangiovanni, G. Kresse, A. Toschi and K. Held, Comparing quasiparticle $G W+D M F T$ and $L D A+D M F T$ for the test bed material SrVO3, Physical Review B 88(16), 165119 (2013), doi:10.1103/physrevb.88.165119.

[31] K. Yoshimi, J. Otsuki, Y. Motoyama, M. Ohzeki and H. Shinaoka, SpM: Sparse modeling tool for analytic continuation of imaginary-time Green's function, Computer Physics Communications 244, 319 (2019), doi:10.1016/j.cpc.2019.07.001.

[32] R. Levy, J. LeBlanc and E. Gull, Implementation of the maximum entropy method for analytic continuation, Computer Physics Communications 215, 149 (2017), doi:https://doi.org/10.1016/j.cpc.2017.01.018.

[33] H. Shinaoka, D. Geffroy, M. Wallerberger, J. Otsuki, K. Yoshimi, E. Gull and J. Kuneš, Sparse sampling and tensor network representation of two-particle Green's functions, SciPost Physics 8(1), 012 (2020), doi 10.21468/scipostphys.8.1.012.

[34] L. Huang, i QIST v0.\%: An open source continuous-time quantum Monte Carlo impurity solver toolkit, Computer Physics Communications 221, 423 (2017), doi:10.1016/j.cpc.2017.08.026, 1708.07453. 